Accepted to the Astrophysical Journal

Preprint typeset using $\mathrm{LAT}_{\mathrm{E} X} \mathrm{X}$ style emulateapj v. 12/16/11

\title{
THE ATMOSPHERIC CIRCULATION OF A NINE-HOT JUPITER SAMPLE: PROBING CIRCULATION AND CHEMISTRY OVER A WIDE PHASE SPACE
}

\author{
Tiffany Kataria ${ }^{1}$, David K. Sing ${ }^{1}$, Nikole K. Lewis ${ }^{2}$, Channon Visscher $^{3}$, Adam P. Showman ${ }^{4}$, Jonathan J. \\ FORTNEY $^{5}$, MARK S. MARLEY ${ }^{6}$ \\ Accepted to the Astrophysical Journal
}

\begin{abstract}
We present results from an atmospheric circulation study of nine hot Jupiters that comprise a large transmission spectral survey using the Hubble and Spitzer Space Telescopes. These observations exhibit a range of spectral behavior over optical and infrared wavelengths which suggest diverse cloud and haze properties in their atmospheres. By utilizing the specific system parameters for each planet, we naturally probe a wide phase space in planet radius, gravity, orbital period, and equilibrium temperature. First, we show that our model "grid" recovers trends shown in traditional parametric studies of hot Jupiters, particularly equatorial superrotation and increased day-night temperature contrast with increasing equilibrium temperature. We show how spatial temperature variations, particularly between the dayside and nightside and west and east terminators, can vary by hundreds of $\mathrm{K}$, which could imply large variations in $\mathrm{Na}, \mathrm{K}, \mathrm{CO}$ and $\mathrm{CH}_{4}$ abundances in those regions. These chemical variations can be large enough to be observed in transmission with high-resolution spectrographs, such as ESPRESSO on VLT, METIS on the E-ELT, or with MIRI and NIRSpec aboard JWST. We also compare theoretical emission spectra generated from our models to available Spitzer eclipse depths for each planet, and find that the outputs from our solar-metallicity, cloud-free models generally provide a good match to many of the datasets, even without additional model tuning. Although these models are cloud-free, we can use their results to understand the chemistry and dynamics that drive cloud formation in their atmospheres.
\end{abstract}

Keywords: planets and satellites: general, methods: numerical, atmospheric effects

\section{INTRODUCTION}

After nearly two decades of milestone discoveries in exoplanet science, current and future observational efforts seek to discover and characterize smaller and cooler planets at larger orbital distances. Even so, the close-in, tidally-locked "hot Jupiters" remain an important population for exoplanet characterization-they are still the best targets for probing atmospheric properties, refining observational techniques, and expanding current theory, all of which can be extended to smaller planets.

Because many hot Jupiters transit their host stars, observations during transit (when the planet passes in front of its star) through secondary eclipse (when the planet passes behind its star) have allowed us to characterize their atmospheres. Already, dozens of hot Jupiters have been observed with the Spitzer Space Telescope, Hubble Space Telescope (HST), and many ground-based facilities (e.g., Charbonneau et al. 2002; Deming et al. 2005; Redfield et al. 2008). Our insights will only deepen with the advent of eclipse mapping, which can place constraints on both the longitudinal and latitudinal temperature structure (e.g., de Wit et al. 2012; Nikolov \&

\footnotetext{
${ }^{1}$ Astrophysics Group, School of Physics, University of Exeter, Stocker Road, Exeter EX4 4QL, UK; tkataria@astro.ex.ac.uk

${ }^{2}$ Space Telescope Science Institute, 3700 San Martin Drive, Baltimore, MD 21218, USA

${ }^{3}$ Department of Chemistry, Dordt College, Sioux Center, Iowa 51250, USA

${ }^{4}$ Department of Planetary Sciences and Lunar and Planetary Laboratory, The University of Arizona, Tucson, AZ 85721, USA

${ }^{5}$ Department of Astronomy \& Astrophysics, University of California, Santa Cruz, CA 95064, USA

${ }^{6}$ NASA Ames Research Center 245-3, Moffett Field, CA 94035, USA
}

Sainsbury-Martinez 2015). Despite occupying a small region of exoplanet parameter space, these observations have shown that hot Jupiters exhibit a wide diversity in spectral properties and atmospheric compositions.

Recently, Sing et al. (2016) presented an ensemble of transmission spectra from a large HST observational program of ten hot Jupiters (HD 189733b, HD 209458b, WASP-6b, WASP-12b, WASP-17b, WASP-19b, WASP31b, WASP-39b, HAT-P-1b, and HAT-P-12b) using STIS and WFC3, as well as the Spitzer 3.6 and 4.5 $\mu \mathrm{m}$ IRAC channels (HST GO-12473; P.I. Sing). Their spectra show a range of water and alkali abundances, as well as Rayleigh scattering slopes at near-UV and optical wavelengths, that indicate a diversity in cloud and haze properties (see also Pont et al. 2013; Sing et al. 2013; Huitson et al. 2013; Wakeford et al. 2013; Nikolov et al. 2014, 2015; Sing et al. 2015). More importantly, the water abundance for each planet correlates with atmospheric type, suggesting that hot Jupiters are not depleted in primordial water during formation, but simply that clouds and hazes obscure its spectral feature.

General circulation models (GCMs) are useful tools for understanding the nature of these clouds and hazes, as the three-dimensional wind and temperature structure sets their formation and transport. Here we present cloud-free GCMs coupled to a nongray radiative transfer scheme for each Large Program (LP) planet. Unlike other parametric circulation studies of hot Jupiters, which typically model one or two individual planets, this study is the first of its kind to compare circulation models for nine individual planets. Such a large ensemble of hot Jupiters naturally allows for a comparative study over a wide parameter space in planet radius, gravity, 
Table 1

Planetary and stellar parameters of the HST Large Program (LP) planets modeled in our study. See main text for definitions of columns 8-12.

\begin{tabular}{|c|c|c|c|c|c|c|c|c|c|c|c|}
\hline Planet & $\begin{array}{c}\mathrm{R}_{\mathrm{p}} \\
\left(\mathrm{R}_{\mathrm{J}}\right)\end{array}$ & $\begin{array}{c}T_{\text {orb }}=T_{\text {rot }} \\
\text { (Earth days) }\end{array}$ & $\begin{array}{c}\Omega \\
\left(10^{-5} \mathrm{~s}^{-1}\right)\end{array}$ & $\begin{array}{c}g \\
\left(\mathrm{~m} \mathrm{~s}^{-2}\right)\end{array}$ & $\begin{array}{l}T_{e q} \\
(\mathrm{~K})\end{array}$ & $\begin{array}{c}F_{\star} \\
\left(\mathrm{W} \mathrm{m}^{-2}\right)\end{array}$ & $\begin{array}{c}H \\
(\mathrm{~km})\end{array}$ & $\overline{R_{o}}$ & $N_{\text {jets }}$ & $\begin{array}{c}L_{D} \\
\left(\times 10^{7} \mathrm{~m}\right)\end{array}$ & $\begin{array}{c}L_{\beta} \\
(\mathrm{km})\end{array}$ \\
\hline HAT-P-12b & 0.96 & 3.21 & 2.263 & 5.62 & 963 & $1.917 \mathrm{e} 5$ & 648 & 0.82 & 1.28 & 5.4 & 16.7 \\
\hline WASP-39b & 1.27 & 4.06 & 1.793 & 4.07 & 1117 & $3.548 \mathrm{e} 5$ & 1037 & 0.92 & 1.35 & 6.5 & 20.3 \\
\hline WASP-6b & 1.22 & 3.36 & 2.164 & 8.71 & 1145 & $3.452 \mathrm{e} 5$ & 497 & 0.89 & 1.26 & 7.2 & 19.6 \\
\hline HD $189733 b$ & 1.14 & 2.22 & 3.279 & 21.40 & 1201 & $4.632 \mathrm{e} 5$ & 212 & 0.67 & 1.59 & 5.1 & 16.4 \\
\hline HAT-P-1b & 1.32 & 4.46 & 1.631 & 7.46 & 1322 & $7.016 \mathrm{e} 5$ & 670 & 1.32 & 1.17 & 8.0 & 24.8 \\
\hline HD $209458 b$ & 1.36 & 3.52 & 2.066 & 9.35 & 1448 & $1.001 \mathrm{e} 6$ & 585 & 1.26 & 1.31 & 7.4 & 24.6 \\
\hline WASP-31b & 1.55 & 3.40 & 2.139 & 4.56 & 1575 & $1.399 \mathrm{e} 6$ & 1305 & 1.06 & 1.40 & 7.9 & 24.0 \\
\hline WASP-17b & 1.89 & 3.73 & 1.950 & 3.57 & 1738 & $2.140 \mathrm{e} 6$ & 1840 & 1.09 & 1.43 & 9.4 & 26.9 \\
\hline WASP-19b & 1.41 & 0.79 & 9.217 & 14.21 & 2050 & $4.392 \mathrm{e} 6$ & 545 & 0.32 & 2.59 & 3.9 & 12.5 \\
\hline
\end{tabular}

Note. - The values for $R_{p}, T_{o r b}, T_{r o t}, \Omega, g, T_{e q}, F_{\star}$ and $H$ were adopted from values in Sing et al. (2016) and references therein. The values for $R_{o}, N_{j e t s}, L_{D}$, and $L_{\beta}$ were derived from GCM outputs. See text for more details.

orbital period, and equilibrium temperature.

We should stress that the primary focus of this paper is not to provide a detailed analysis of the transmission spectra themselves. Rather, this paper serves as the first step to interpreting the ensemble of transmission spectra by exploring each planet's three-dimensional atmospheric temperature structure and dynamics, which set the formation of the clouds and hazes that are inferred from the data. We do, however, compare our models to observed dayside thermal emission observations, as those datasets are directly related to each planet's thermal structure. Furthermore, we stress that the main objective of this study is not to analyze each individual planet on its own; rather, we aim to use this "grid" of planets to probe trends in dynamics, temperature, and chemistry across the entire sample. In Section 2 we describe our model setup, the SPARC/MITgcm. In Section 3 we present the major results of our comparative study. Section 4 provides a discussion of these results, and we conclude in Section 5.

\section{MODEL SETUP}

To model each planet's atmospheric circulation, we utilize the Substellar and Planetary Radiation and Circulation (SPARC) model (Showman et al. 2009). The SPARC model couples the general circulation model maintained at the Massachusetts Institute of Technology (the MITgcm, Adcroft et al. 2004) with a planeparallel, two-stream version of the multi-stream radiation code developed by Marley \& McKay (1999). The MITgcm is a finite-volume code that solves the threedimensional primitive equations on a staggered Arakawa C grid (Arakawa \& Lamb 1977). We use a curvilinear coordinate system called the "cubed-sphere"; unlike a traditional latitude-longitude grid, the cubed-sphere lacks singularities at the poles, allowing for longer timesteps throughout the domain. We maintain numerical stability using a fourth-order Shapiro filter on the time derivatives of the wind and temperature at each timestep; this smooths the grid-scale variations while minimally affecting the flow at large scales.

The radiative transfer (RT) code solves the two-stream radiative transfer equations and employs the correlated$k$ method (Goody et al. 1989; Marley \& McKay 1999) over 11 spectral bins (Kataria et al. 2013), which retains most of the accuracy of full line-by-line calculations while drastically increasing computational efficiency. Opaci- ties are calculated assuming local thermodynamic and chemical equilibrium for each pressure-temperature $(p$ T) point, using the solar photospheric elemental abundances of Lodders (2003). The coupling of the dynamical core and radiative transfer scheme allow for the selfconsistent calculation of the heating and cooling rates of the atmosphere. At each grid point, the MITgcm calculates the wind and temperature fields, which are used by the RT scheme to calculate the upward and downward fluxes at each pressure layer. These fluxes are used to update the heating and cooling rates, which are then used by the MITgcm.

The SPARC model is already fully operational and has been extensively used for hot Jupiters (Showman et al. 2009; Kataria et al. 2013; Parmentier et al. 2013; Showman et al. 2013; Lewis et al. 2014; Kataria et al. 2015; Showman et al. 2015), sub-Neptunes (Lewis et al. 2010), and super Earths (Kataria et al. 2014). We derive our input spectrum for each parent star from the PHOENIX stellar atmosphere models (Hauschildt et al. 1999).

We focus our modelling on nine of the ten individual planets that comprise the LP sample. Their system parameters are listed in Table 1 . We leave a detailed study of the circulation of WASP-12b as future work, noting that with its large equilibrium temperature $(\sim 2500 \mathrm{~K})$, it is far out of the parameter space probed in this study. Table 1 also includes values that help diagnose the dynamics (columns 8-12). We briefly discuss them here; for a more complete discussion of the parameters and their interpretation, see Showman et al. (2010).

First, we calculate each planet's pressure scale height, $H$, which is generally defined as

$$
H=\frac{k T}{m g}
$$

where $k$ is the Stefan-Boltzmann constant, $T$ is the temperature, $m$ is the mean-molecular weight and $g$ is the planetary gravity. Here, we calculate a reference scale height using the equilibrium temperature, $T_{e q}$. The Rossby number, $R_{o}$, is a dimensionless parameter which diagnoses the importance of rotation on the dynamics. It is defined as

$$
R_{o}=\frac{U}{f L}
$$

where $U$ is a characteristic wind speed, $f$ is the Corio- 

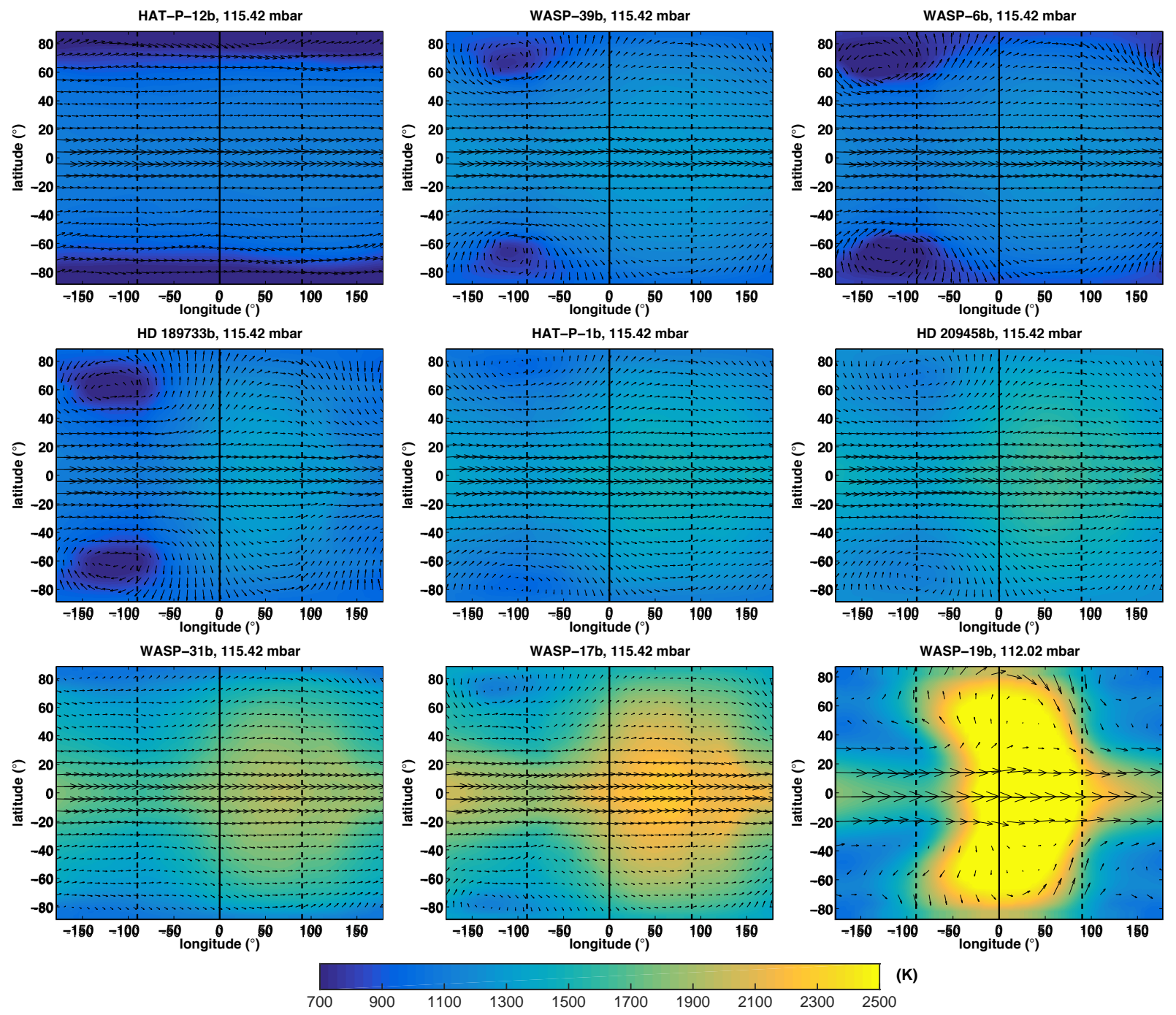

Figure 1. Wind/temperature profiles for each LP target at a pressure of $100 \mathrm{mbar}$. Panels are organized by increasing planetary equilibrium temperature, $T_{e q}$, and are plotted on the same colorscale. Each vertical solid line denotes the substellar longitude, while dashed vertical lines denote the western and eastern terminators, respectively.

lis parameter, $2 \Omega \sin \phi$, where $\phi$ is the latitude, and $L$ is a characteristic length scale (in this case, the scale height, $H)$. We assume the characteristic wind speed to be the root-mean squared (RMS) velocity ${ }^{7}$ at 100 mbar from our simulations, approximately the level of the infrared (IR) photosphere. We also calculate two dynamical length scales for each planet: the equatorial deformation radius, $L_{D}$, which describes the characteristic length scale over which the atmosphere geostrophically adjusts to large-scale phenomena, and the Rhines scale, $L_{\beta}$, which defines the length scale over which a flow transitions to zonally-banded flow (that is, the length scale over which the flow reorganizes into jets)(Holton 1992). At the equator, these are defined as

$$
L_{D}=\left(\frac{N H}{\beta}\right)^{1 / 2}
$$

7 the RMS velocity was calculated as prescribed in Lewis et al. (2010), $V_{r m s}(p)=\sqrt{\frac{\int\left(u^{2}+v^{2}\right) d A}{A}}$, where $u$ is the zonal velocity and $v$ is the meridional velocity. and

$$
L_{\beta}=\pi\left(\frac{U}{\beta}\right)^{1 / 2}
$$

where $N$ is the Brunt-Väisälä frequency, and $\beta=d f / d y$ is the beta parameter, the variation in the Coriolis force with latitude. At the equator, $\beta=2 \Omega \cos \phi / \mathrm{R}_{\mathrm{p}}$, where $R_{p}$ is the planet radius.

Showman \& Polvani (2011) have shown that the equatorial jet width should be set by $L_{D}$. We can then use our formulation of $L_{D}$ to derive an expression for the number of jets expected in each atmosphere by dividing the planet radius by the deformation radius,

$$
N_{j e t s} \sim\left(\frac{2 \Omega \mathrm{R}_{\mathrm{p}}}{N H}\right)^{1 / 2}
$$

We also include each planet's orbital period, $T_{\text {orb }}$, and rotation period, $T_{\text {rot }}$, which are assumed to be equal (i.e., synchronous rotation).

For all planet models, we assume an atmospheric metallicity of $1 \times$ solar. Models for planets cooler than WASP-19b have a horizontal resolution of C32 $(128 \times 64$ 
in longitude and latitude, respectively) and a vertical resolution of 53 pressure levels, evenly spaced in log pressure, that extend from a mean pressure of 200 bars at the bottom to $2 \mu \mathrm{bar}$ at the top. We run the circulation model of WASP-19b at lower resolution to maintain numerical stability; it has a horizontal resolution of $\mathrm{C} 16$ $(63 \times 32)$ and a vertical resolution of 46 pressure levels, evenly spaced in log pressure from a mean pressure of 1000 bars at the bottom to 0.2 mbar at the top. We have shown in a previous study (Kataria et al. 2015) that low resolution models still capture the bulk dynamical structure of hot Jupiters; therefore, the model of WASP-19b is sufficient for our comparison study.

\section{RESULTS}

\subsection{Circulation regime}

Previous parametric studies (e.g., Perez-Becker \& Showman 2013; Showman et al. 2015) have shown that with increasing stellar irradiation, the atmospheric circulation of hot Jupiters transitions from a regime that is dominated by banded zonal flow with small day-night temperature contrasts, to a regime with strong day-night flow and large day-night contrasts ${ }^{8}$. We recover this trend in our "grid" of models for each individual planet when we sort their wind/temperature profiles and zonalmean zonal wind profiles by increasing equilibrium temperature, $T_{e q}$ (Figures 1 and 2). When comparing the coolest hot Jupiter in our sample, HAT-P-12b, with the hottest planet in our sample, WASP-19b, at a given pressure of 100 mbar (within the range of observable pressures), HAT-P-12b exhibits little to no day-night temperature variation $(<50 \mathrm{~K})$ with weak zonal flow, while WASP-19b has a very large temperature contrast $(\sim 800$ 1000 K, Figure 1) and strong equatorial flow.

Despite these differences in day-night temperature structure, the prevailing wind direction at photospheric pressures is eastward for all planets in our sample, a consequence of each planet's equatorial superrotation (Figure 2). This superrotation arises from the large daynight forcing each planet receives throughout its orbit, which excite Rossby and Kelvin waves and induce eddy phase tilts that interact with the mean flow to transport eastward angular momentum to the equator (Showman \& Polvani 2011). This produces the chevron-shaped hotspot seen in wind and temperature profiles of HD 189733b, HAT-P-1b, HD 209458b, WASP-31b, WASP$17 \mathrm{~b}$, and WASP-19b (Figure 1). The peak speeds of the equatorial jets increase with increasing $T_{e q}$, from $\sim 3 \mathrm{~km} \mathrm{~s}^{-1}$ for HAT-P-12b to $\sim 6 \mathrm{~km} \mathrm{~s}^{-1}$ for WASP19b. Furthermore, each wind/temperature map shows that the western terminator is cooler than the eastern terminator (left and right dashed lines, respectively), which could imply different chemical properties across each limb (see Section 3.3).

Interestingly, HAT-P-12b, HD 189733b and WASP-19b each have multiple jets in their atmospheres. In addition to an equatorial jet, HAT-P-12b has two eastward jets at high latitudes $\left( \pm 80^{\circ}\right)$ with speeds exceeding $1 \mathrm{~km} \mathrm{~s}^{-1}$, while HD 189733b has two westward jets at mid-latitudes

\footnotetext{
8 This trend exists even in the absence of friction, which would further inhibit day-night flow and also lead to large day-night contrasts. The circulation models presented here do not include drag, and we therefore do not discuss their effects in further detail.
}

$\left( \pm 60^{\circ}\right)$ with speeds of $600 \mathrm{~m} \mathrm{~s}^{-1}$. WASP-19b has both high-latitude $\left( \pm 80^{\circ}\right)$ eastward jets exceeding $1 \mathrm{~km} \mathrm{~s}^{-1}$ and mid-latitude $\left( \pm 50^{\circ}\right)$ westward jets exceeding 200 $\mathrm{m} \mathrm{s}^{-1}$. This is a result of each planet's comparatively fast rotation rate and small Rossby deformation radius (Table 1), which allow for the formation of multiple jets. The effect of rotation rate and orbital distance (and similarly, stellar flux, $F_{\star}$ ) on atmospheric circulation has been explored in a number of parametric studies of hot Jupiters (Kataria et al. 2013; Showman et al. 2015). In particular, Showman et al. (2015) explore planets over a wide range of stellar fluxes and rotation rates, from "cold" to "hot" $\left(1.16 \times 10^{4}\right.$ to $\left.4.65 \times 10^{5} \mathrm{~W} \mathrm{~m}^{-2}\right)$ and from "slow" to "fast" $\left(8.264 \times 10^{-6}\right.$ to $\left.1.322 \times 10^{-4} \mathrm{~s}^{-1}\right)$. While this study does not probe as large a parameter space, we can place our model results for these three planets in the context of Showman et al. (2015). HD 189733b is identical to the "H $\Omega_{\text {med" }}$ case, while HAT-P-12b has a similar rotation rate to HD $189733 \mathrm{~b}$, but a smaller stellar insolation (Table 1). Therefore, HAT-P-12b falls in the phase space between the "hot" and "warm" moderately fast rotating hot Jupiters, where the flow transitions from superrotation at the equator to the high latitudes (see Figs. 3 and 4 in Showman et al. 2015, second/third rows, middle panels). WASP-19b is both highly irradiated and a fast rotator, and therefore its atmosphere forms multiple high latitude jets.

\subsection{Three-dimensional temperature considerations}

While transiting planets offer many observational strategies by which we can characterize their atmospheres, one should be careful to consider that each technique probes different spatial regions of the planet, from the planet's terminator or limb during transit, to the planet's dayside during secondary eclipse, to timevarying longitudes for full phase curve observations. The temperatures probed by each geometry can vary by hundreds of Kelvin, particularly across hemispheres (from dayside to nightside) or even across each limb (from the colder, west terminator, to the hotter, eastern terminator; see Fig.1). Furthermore, transit observations probe shallower pressures than eclipse observations, and phase curves and eclipse maps can probe a range of pressures in a single dataset.

These considerations are important when inferring molecular abundances and cloud or haze properties for an individual planet or a range of planets. For example, the water abundance retrieved from a transmission spectrum could be largely different in an emission spectrum, as they probe different longitudes, temperatures and pressures. Cloud properties are also expected to vary across the planet with temperature, and contribute varying amounts to each spectrum. Therefore, the threedimensional temperature structure must be considered when interpreting an ensemble of hot Jupiter observations, particularly when inferring molecular abundances and cloud properties. Here we use models from our nineplanet study to illustrate the spatial temperature variations that should be considered in emission and transmission observations.

\subsubsection{Emission observations: temperature variations with} longitude 

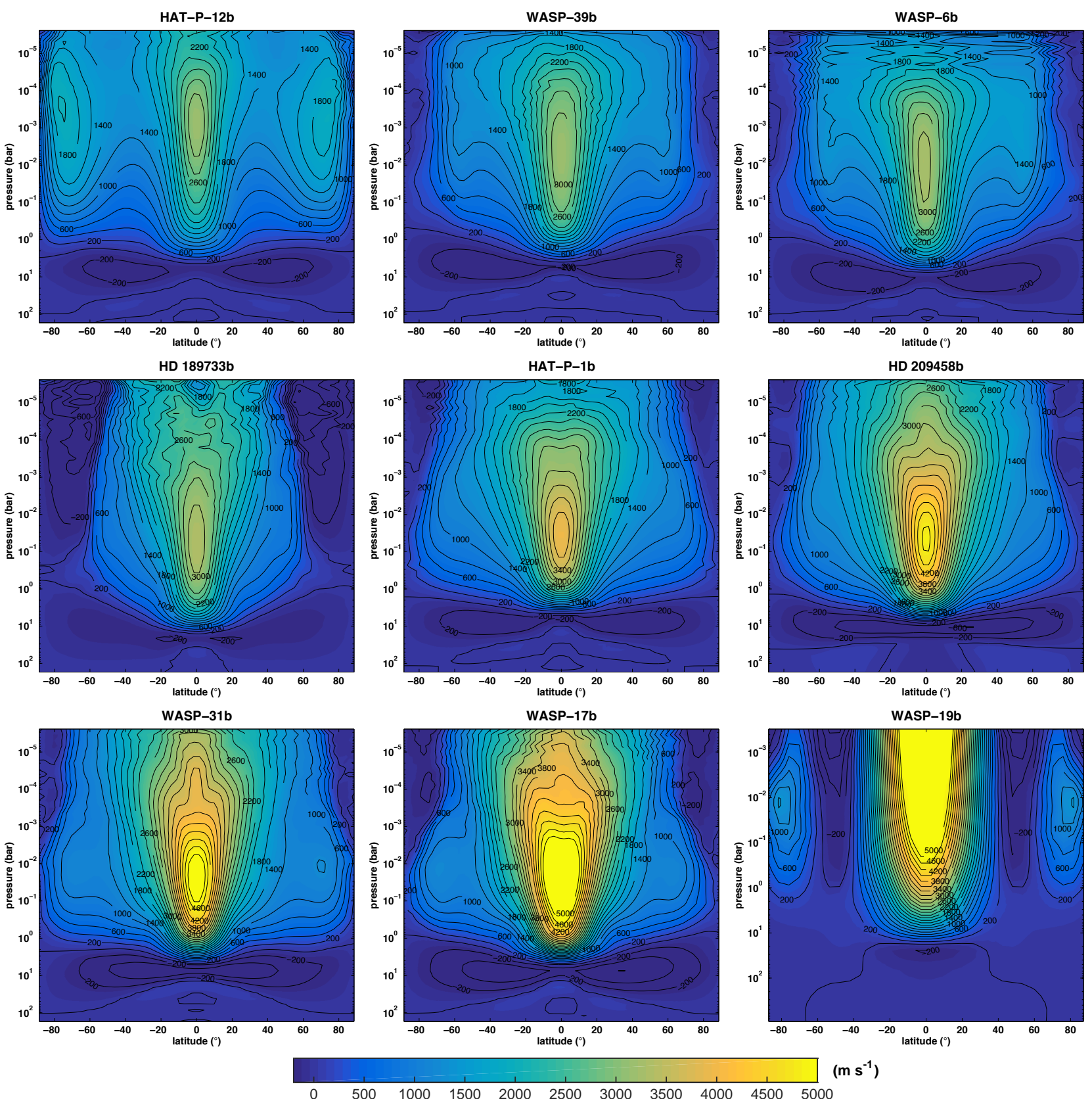

Figure 2. Zonal-mean zonal wind for each individual LP target . Panels are organized by increasing planetary equilibrium temperature, $T_{e q}$, and are plotted on the same colorscale.

When observing the thermal emission from a transiting exoplanet, the contribution of flux from the equator is greater than the contribution from the poles, so we can plot the temperature weighted by the cosine of the planet latitude, $\phi$ for insights to those observations. This is shown in Fig. 3 for each LP planet as a function of longitude and pressure, all plotted on the same colorscale. At a given pressure, we again recover the trend of increasing day-night temperature contrast with increasing $T_{e q}$, as seen in Fig. 1. Furthermore, for cooler hot Jupiters HAT-P-12b, WASP-39b and WASP-6b, the hotspot is shifted approximately $\sim 40-50$ degrees eastward from the substellar point at the range of pressures probed in emission ( 1 bar to 10 mbar; black dashed horizontal lines), while the hotter planets HD 189733b, HAT-P-1b, HD 209458b, WASP-31b, WASP-17b, and WASP-19b have hotspot shifts of $\sim 20-30$ degrees.

We can use these plots as a qualitative basis for predicting the behavior of their IR phase curves. First, we can infer that amplitudes of IR flux will likely be largest and peak IR fluxes will occur closest to secondary eclipse for the hottest planets (e.g., WASP-19b, WASP-17b), while cooler planets will likely exhibit smaller amplitudes and IR fluxes that peak well before secondary eclipse. While we do not include the theoretical phase curves for each planet in this paper, we do include a table of measured phase offsets and flux amplitudes derived from our models at the Spitzer IRAC 3.6 and $4.5 \mu \mathrm{m}$ bandpasses and the HST/WFC3 G141 bandpass (1.12-1.65 $\mu \mathrm{m})$ (Table $2)$. Phase offsets are measured in units of phase relative 

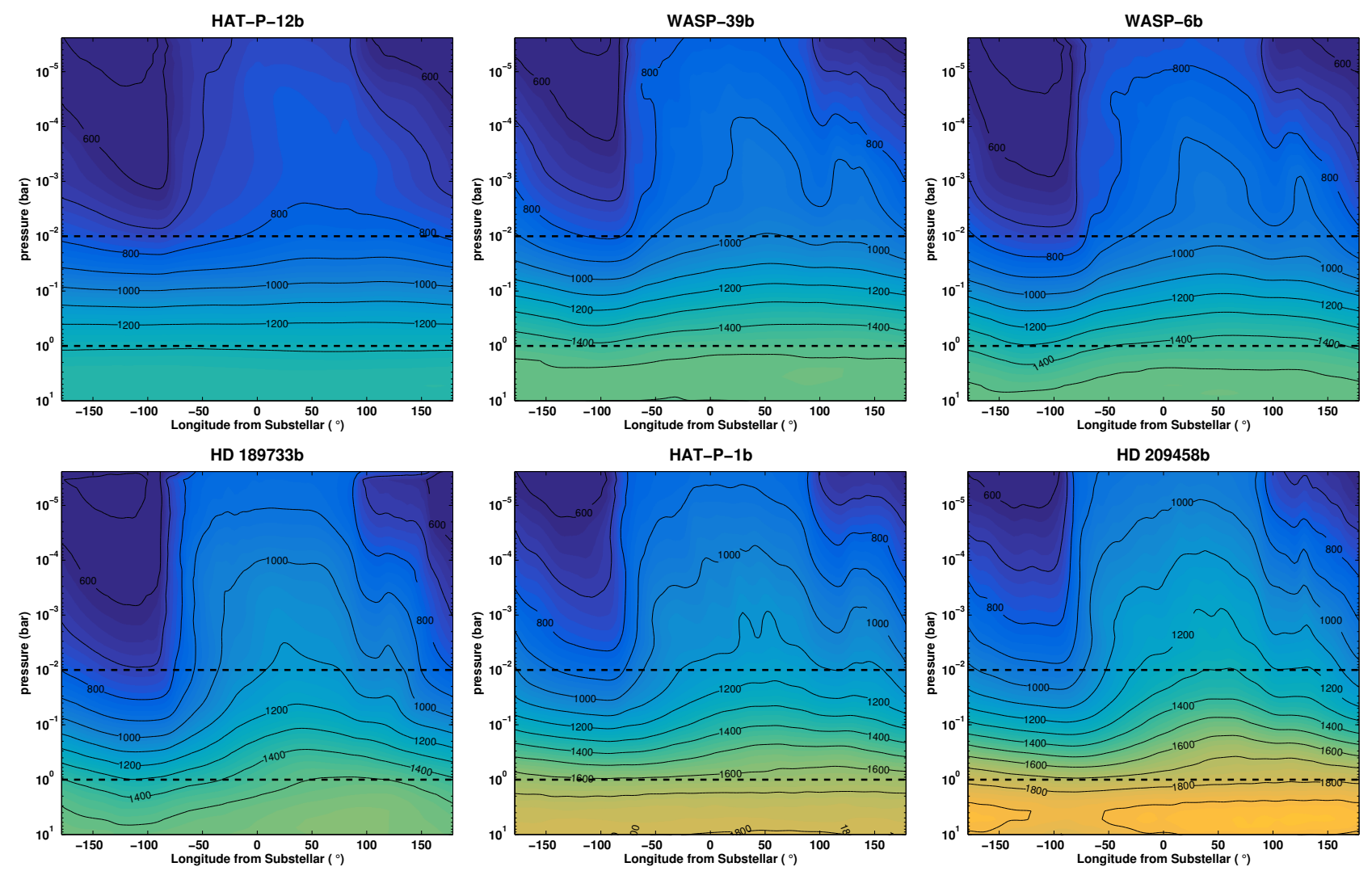

WASP-31b
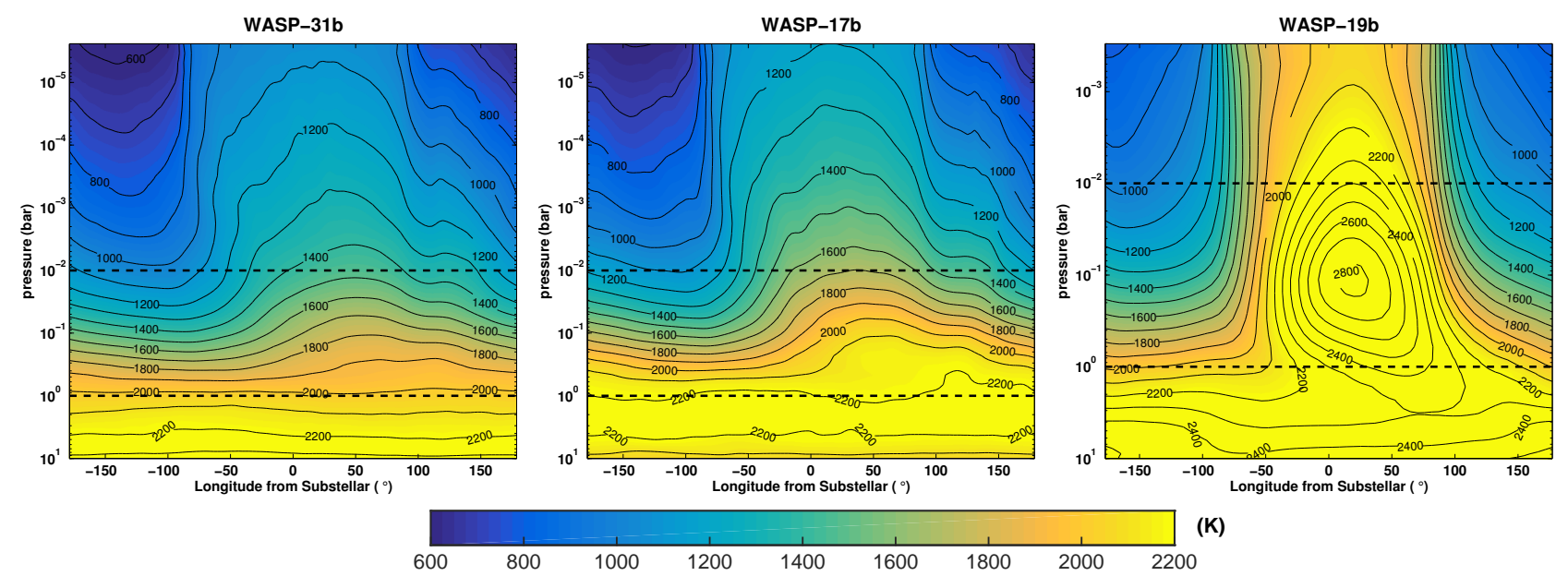

$\frac{1}{1000}$

$\begin{array}{cccccc}1200 & 1400 & 1600 & 1800 & 2000 & 2200\end{array}$

Figure 3. Latitudinally-averaged temperature as a function of pressure and longitude from the substellar longitude for each individual LP planet. Panels are organized by increasing planetary equilibrium temperature, $T_{e q}$, and are plotted on the same colorscale. These average temperatures are weighted by $\cos \phi$, where $\phi$ is latitude; this is equivalent to weighting each grid point by its projection angle toward an observer at the equator. Black dashed lines highlight the pressures typically probed in emission.

to secondary eclipse, where secondary eclipse occurs at a phase of 0.5 . At each bandpass, the timing of peak IR flux offset generally becomes shorter and the phase amplitude generally becomes larger with increasing $T_{e q}$. This is likely because radiative timescales for these hotter planets are much shorter than the timescales for Kelvin and Rossby waves to propagate, which likely suppress the formation of zonal jets (e.g., Showman et al. 2013; Komacek \& Showman 2016). However, chemistry also likely plays a role (Zellem et al. 2014). We also note that our model phase curves have previously been compared to Spitzer phase curves of HD 209458b and HD 189733b (Knutson et al. 2012; Zellem et al. 2014), and future work will also present comparisons to Spitzer 3.6 and $4.5 \mu \mathrm{m}$ phase curves of WASP-19b (Wong et al. 2015a). We also compare theoretical dayside emission spectra to Spitzer observations in Section 3.4.

\subsubsection{Transmission observations: temperature variations with latitude}

Unlike emission, where the observed flux is weighted towards the equator, all latitudes have equal weighting on transit observations. Because we are observing only the limb, we can instead investigate the variations in limb-averaged temperature with latitude. These maps are shown for each LP planet in Figure 4 as a function of pressure and latitude. The limb temperature is cal- 

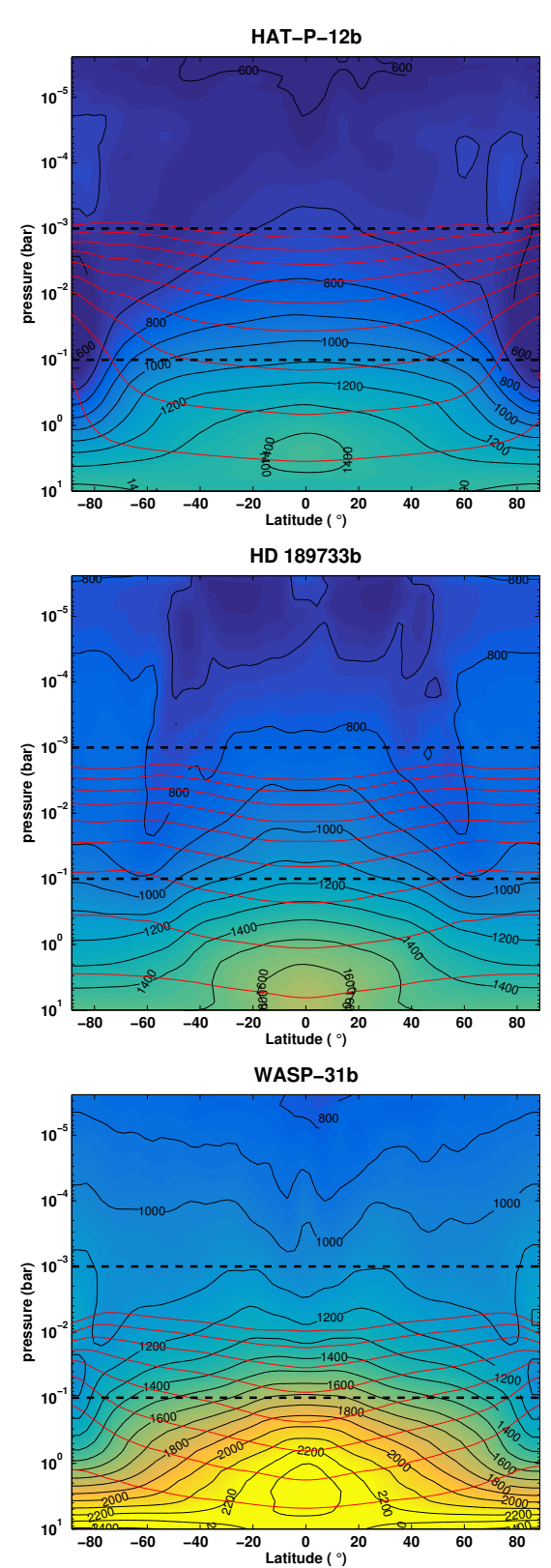

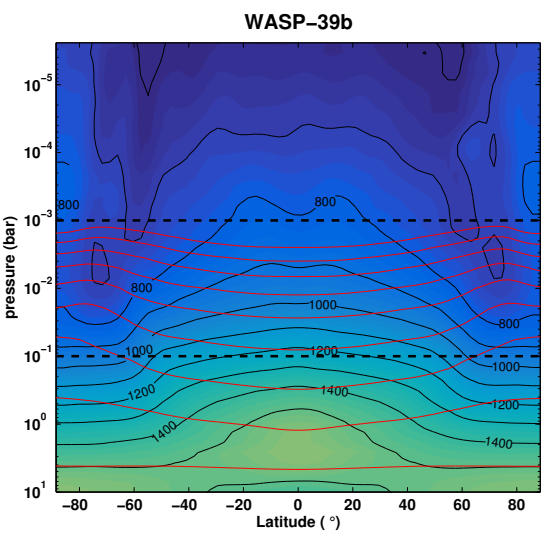

HAT-P-1b

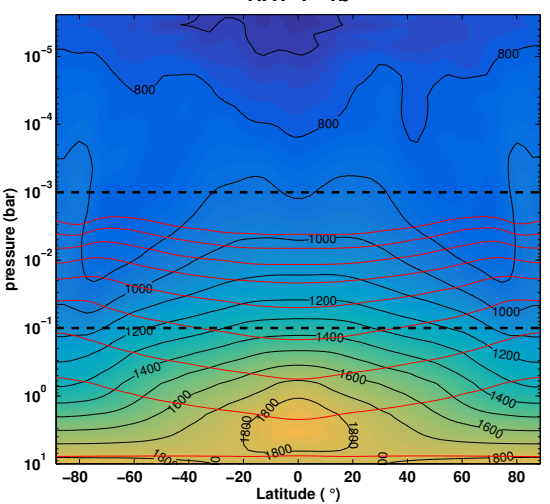

WASP-17b

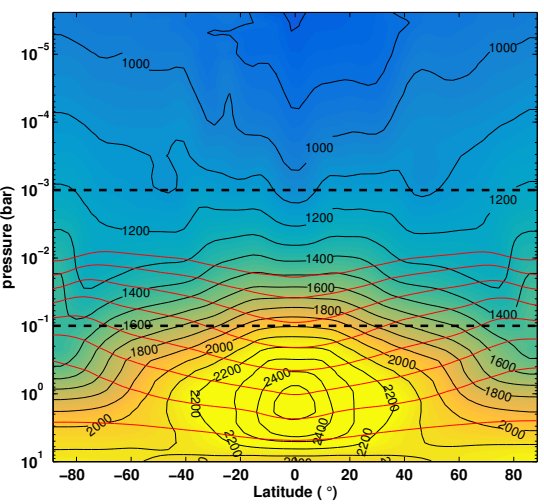

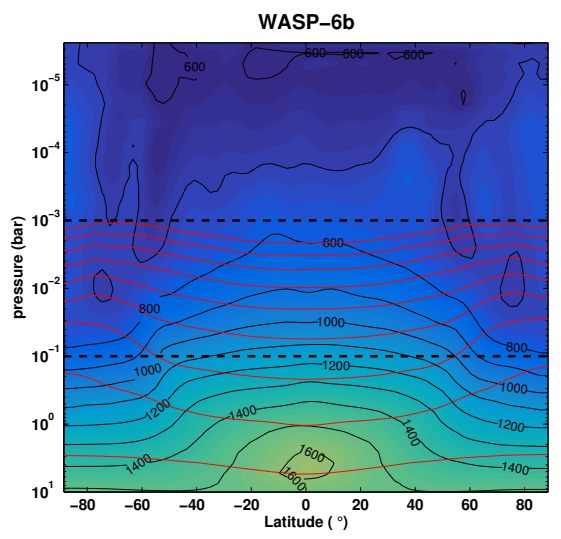

HD 209458b

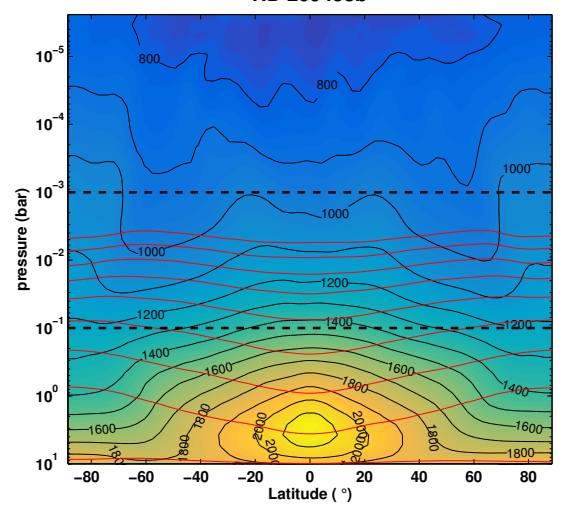

WASP-19b

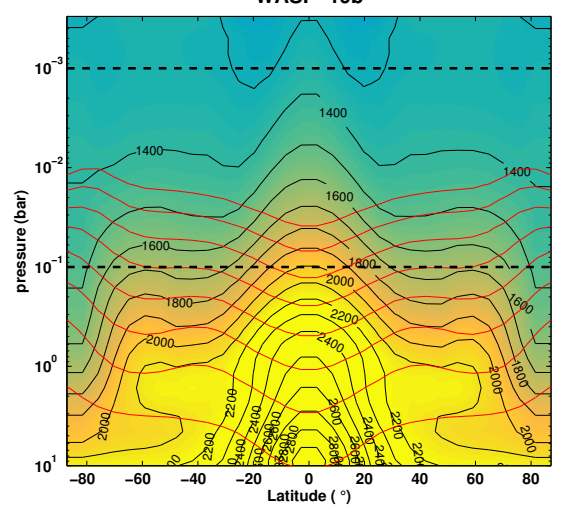

(K)

Figure 4. Limb-averaged temperature as a function of latitude and pressure for each individual LP planet. Panels are organized by increasing planetary equilibrium temperature, $T_{e q}$, and are plotted on the same colorscale. These limb-averaged temperatures are calculated by weighting each grid point that is located along the limb by their grid cell length. Black dashed lines indicate the pressures typically probed in transmission, while red solid lines are isentopes (lines of constant entropy).

culated by weighting each grid point located along the limb by their corresponding grid cell length. For each planet, the hottest regions are confined to the lowest latitudes (less than approximately $\pm 30^{\circ}$ ), and maximum temperatures increase with increasing $T_{e q}$. If we focus on the pressure range probed in transmission $(\sim 100-1$ mbar; black dashed horizontal lines), we see that at a given pressure, temperature variations from the equator to pole can exceed hundreds of $K$ for even the coolest hot Jupiters. On WASP-6b, for example, the temperature at 10 mbar varies from approximately $1200 \mathrm{~K}$ at the equator to approximately $800 \mathrm{~K}$ at the poles, a difference of $\sim 400 \mathrm{~K}$.
The slope of the temperature contours for HAT-P-12b are large, consistent with the large equator-to-pole temperature differences seen in Fig. 1. Coincident with these sloping contours are sloping isentropes (red contours), which indicate constant entropy surfaces. The isentrope slopes of HAT-P-12b become particularly large at high latitudes at pressures of 1 bar to 10 mbar, and suggest that the atmosphere is dynamically unstable (Showman et al. 2015). Indeed, this instability would further explain the high-latitude jets seen in HAT-P-12b's zonalmean zonal wind profile (Fig. 2), as it would lead to development of baroclinic eddies at mid- to high-latitudes, which transport heat meridionally and force and main- 
Table 2

Maximum flux peak offsets and flux amplitudes measured from our theoretical phase curves for each LP planet, in the Spitzer 3.6 and 4.5 $\mu \mathrm{m}$ bandpasses and the HST/WFC3 bandpass $(1.12-1.65 \mu \mathrm{m})$.

\begin{tabular}{|c|c|c|c|c|c|c|}
\hline \multirow[b]{2}{*}{ Planet } & \multicolumn{2}{|c|}{ Spitzer $3.6 \mu \mathrm{m}$} & \multicolumn{2}{|c|}{ Spitzer $4.5 \mu \mathrm{m}$} & \multicolumn{2}{|c|}{ HST/WFC3 $(1.12-1.65 \mu \mathrm{m})$} \\
\hline & Max offset (phase) & Amplitude (ppm) & Max offset (phase) & Amplitude (ppm) & Max offset (phase) & Amplitude (ppm) \\
\hline HAT-P-12b & -0.193 & 138 & -0.220 & 130 & -0.251 & 3.45 \\
\hline WASP-39b & -0.190 & 433 & -0.184 & 332 & -0.218 & 14 \\
\hline HD $189733 b$ & -0.142 & 950 & -0.136 & 821 & -0.181 & 50 \\
\hline HAT-P-1b & -0.186 & 346 & -0.164 & 307 & -0.219 & 16 \\
\hline WASP-31b & -0.169 & 437 & -0.144 & 620 & -0.190 & 57 \\
\hline WASP-17b & -0.137 & 544 & -0.115 & 791 & -0.157 & 110 \\
\hline
\end{tabular}

tain jets at those latitudes. The variably sloping isentropes on WASP-19b suggest baroclinic eddies also play a role in the formation of their high-latitude jets, particularly when coupled with its small deformation radius. Among the rest of the nine-planet sample, the isentrope slopes are comparatively flat, suggesting their atmospheres are comparatively stable. This is especially true for those hot Jupiters that are highly irradiated and slowly rotating, such as WASP-17b and HD 209458b (see Table 1 and Showman et al. 2015).

\subsubsection{Comparing $1 D$ P-T profile averages}

We can summarize the three-dimensional variations in temperature structure for each planet using onedimensional averages, particularly over each hemisphere and limb. Fig. 5 shows the dayside-, nightside-, global, east limb- and west limb-averaged temperature as a function of pressure for each individual planet. Comparing only the dayside- and nightside-averaged profiles (red and yellow profiles, respectively) we see that consistent with our wind and temperature profiles in Figure 1 , the difference between dayside- and nightside-averaged temperature is largest for the hottest planet, WASP-19b, with a difference of $\sim 1000 \mathrm{~K}$ at $1 \mathrm{mbar}$, and smallest for the coolest planet, HAT-P-12b, where the temperature difference is only $\sim 50 \mathrm{~K}$.

When we decompose the limb-averaged profile mapped in Figure 4 to their east and west limb averages (purple and green profiles, respectively), we see that for all nine planets at mbar pressures, the eastern limb is significantly hotter than the west limb. Interestingly, with increasing $T_{e q}$, differences in west and east limb temperatures at photospheric pressures increase then decrease, with WASP-17b, not WASP-19b, having the largest temperature differences $(\sim 300-400 \mathrm{~K})$. This is likely because the increase in overall temperature is mediated by the reduced hotspot offset and increased day-night flow, which begins to homogenize the east- and west-limb temperatures for WASP-19b.

This range of temperature differences between profiles and planets, can imply a diversity in molecular abundances, as well as cloud compositions and cloud base pressures, which will discuss in the following sections. We also note that for all but the hottest planets, the west limb reaches the coolest temperatures, and are actually significantly cooler than even the nightside averages.

\subsection{Chemical implications}

Taken together, the spatial variations in temperature from dayside to nightside, from equator to pole, and also between the east and west terminators, can imply large differences in major molecular abundances. In this section we calculate the mass mixing ratios for seven major species $\left(\mathrm{CH}_{4}, \mathrm{CO}, \mathrm{H}_{2} \mathrm{O}, \mathrm{NH}_{3}, \mathrm{Fe}, \mathrm{Na}\right.$ and $\mathrm{K}$ ) by interpolating the dayside-, nightside-, east- and westterminator-averaged 1D $p$-T profiles from Figure 5 onto a chemical equilibrium abundance $\mathrm{P}-\mathrm{T}$ grid. While we note that the these highly-irradiated atmospheres may not obey local chemical equilibrium (see below), here we assume a pristine environment to explore these abundances.

Figures 6 and 7 plot the mass mixing ratios as a function of pressure. In Figure 6, solid (dashed) profiles signify the dayside (nightside) abundances, while the eastern (western) limb abundances are denoted by the solid (dashed) lines in Figure 7. Rather than focusing on the abundances of each individual planet, we identify trends in abundance variations for each of the species (or groups of species) in Sections 3.3.1-3.3.5.

\subsection{1. $\mathrm{H}_{2} \mathrm{O}$ abundance}

$\mathrm{H}_{2} \mathrm{O}$ is one of the more abundant species in each planet's atmosphere (yellow profiles). Its mixing ratio remains largely unchanged with pressure for each planet in our sample; this is true for the dayside and nightside profiles (Fig.6, yellow solid and dashed profiles, respectively), as well as the east and west limb profiles (Fig. 7, yellow solid and dashed profiles, respectively).

\subsubsection{Fe abundance}

Considering the dayside and nightside profiles in Figure 6 , we would expect that Fe (green profiles) would not be very abundant in the atmospheres of HAT-P-12b, WASP-39b, WASP-6b and HD 189733b, with approximately equal mixing ratios on the dayside and nightside. As the we move to hotter planets in our sample, where Fe is expected to condense on the nightside, Fe abundances on the dayside and nightside can differ by orders of magnitude; this is true for WASP-17b, whose dayside Fe mass mixing ratio is $\sim 10^{-3}$ at all pressures and whose nightside abundance falls from $\sim 10^{-4}$ at 100 mbar to $10^{-10}$ at $\sim 1$ mbar. Comparing the east and west limb abundances (Fig. 6), Fe is more abundant on the eastern limb than the western limb by at least an order of magnitude at pressures probed in transmission (grey box) for all planets except HAT-P-12b, whose $\mathrm{Fe}$ abundance is equally small on both limbs.

\subsection{3. $\mathrm{NH}_{3}$ abundance}



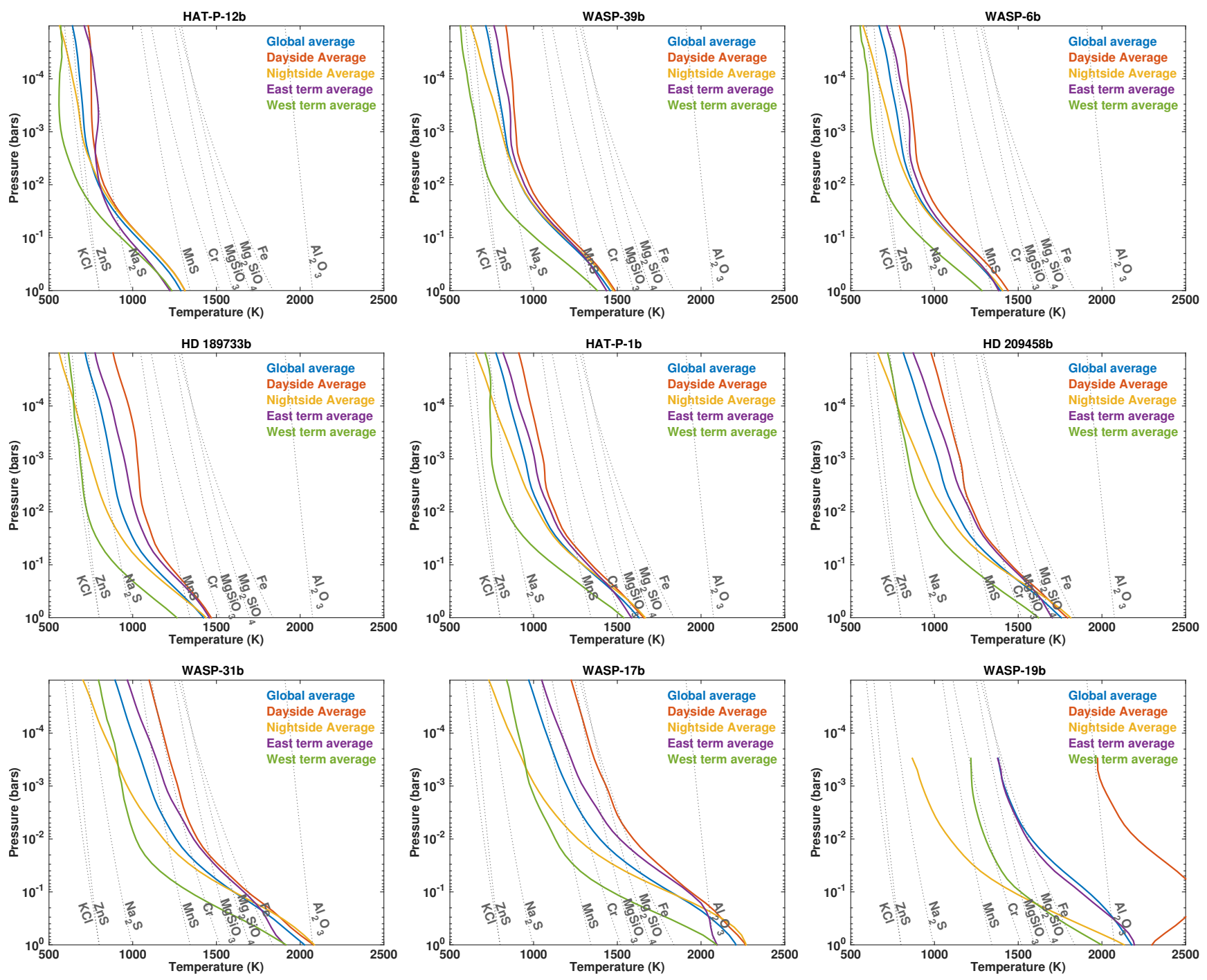

Figure 5. Global- dayside-, nightside-, east terminator- and west terminator-averaged temperature as a function of pressure for each individual LP target. Panels are organized by increasing planetary equilibrium temperature, $T_{e q}$. Condensation curves are also plotted in grey dotted lines.

Comparing the dayside and nightside mass mixing ratios, ammonia abundance does not differ significantly for most planets in our sample (purple profiles). The exception is WASP-19b, where ammonia is less abundant on the dayside than the nightside by 1-2 orders of magnitude at pressures probed in emission. Differences in ammonia abundance are more noticeable when comparing the eastern and western limbs; between 1-100 mbar the variations are about an order of magnitude. However, those variations are actually smallest for WASP-19b.

\subsubsection{Na and $K$ abundance}

Comparing the dayside and nightside mass mixing ratios (Fig. 6), the abundances of $\mathrm{Na}$ and $\mathrm{K}$ (light blue and crimson profiles, respectively) are approximately constant with pressure for planets with $T_{e q} \gtrsim 1300 \mathrm{~K}$ : HATP-1b, HD 209458b, WASP-31b, WASP-17b and WASP$19 \mathrm{~b}$, and do not differ significantly between the dayside and nightside. However, for the four cooler planets with $T_{e q} \lesssim 1200 \mathrm{~K}$ (HAT-P-12b, WASP-39b, WASP-6b, and HD 189733b), Na and K nightside abundances deviate from their dayside abundances at pressures less than $\sim 10$ mbar, where they are depleted by the formation of $\mathrm{Na}_{2} \mathrm{~S}$ and $\mathrm{KCl}$ clouds. In the case of WASP-19b, K is actually more abundant on the nightside; this is because dayside temperatures are high enough for an appreciable fraction of $\mathrm{K}$ to ionize.

Comparing the eastern and western terminator abundances (Figure 7), we see the same general trend, with hotter planets largely abundant in $\mathrm{Na}$ and $\mathrm{K}$ and minimal deviations between east and west limbs. However, HAT-P-1b is among the cooler planets that show significant differences in east/west limb abundances; the western limb $\mathrm{Na}$ and $\mathrm{K}$ abundances differ from the eastern limb by approximately two orders of magnitude at pressures of $\sim 1$ mbar. Planets cooler than HAT-P-1b show deviations of at least four orders of magnitude between east and west limb abundances at pressures less than $\sim 10$ mbar. These cooler planets, then, would be expected to have reduced $\mathrm{Na}$ and $\mathrm{K}$ transmission signals on the western terminator as compared to the comparatively hotter targets, due to the formation of clouds. We further discuss the presence of clouds and the potential to probe these abundance variations in transit in Section 4. 

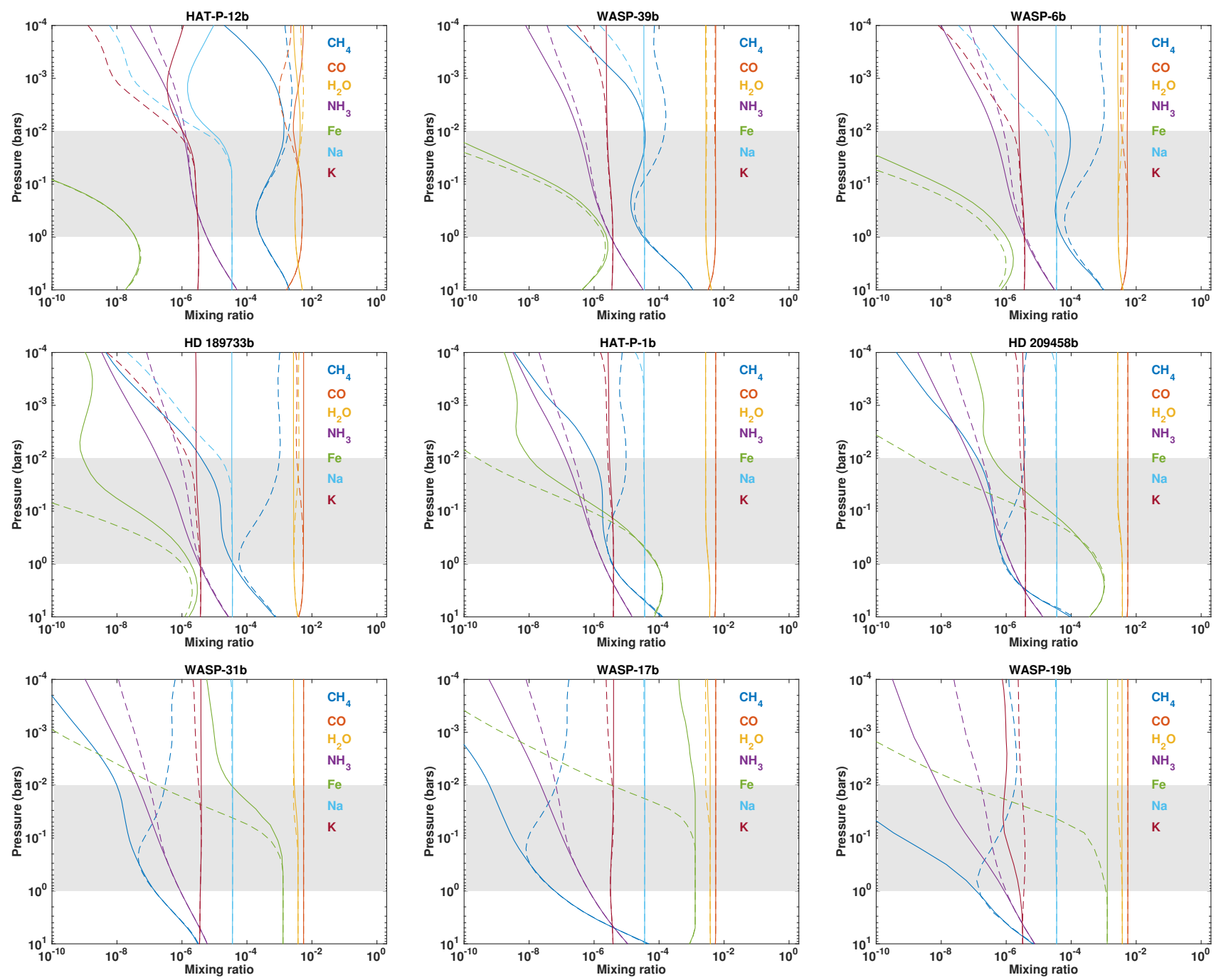

Figure 6. Chemical equilibrium abundances across the dayside (solid profiles) and nightside (dashed profiles) for each $\mathrm{LP}_{\text {planet. }} \mathrm{CH}_{4}$ and $\mathrm{CO}$ abundances are indicated by the thicker orange and blue lines, respectively. Grey boxes indicate the pressures typically probed in emission.

\subsection{5. $\mathrm{CO}$ and $\mathrm{CH}_{4}$ abundance}

This nine-planet sample is especially interesting for comparing $\mathrm{CO}$ and $\mathrm{CH}_{4}$ abundances (orange and blue profiles, respectively), as the intraconversion between these two species occurs over this temperature range, similar to the $\mathrm{L}$ to $\mathrm{T}$ transition for brown dwarfs. If we first consider only the dayside profiles in Figure 6, we see that the dayside $\mathrm{CO}$ varies little with pressure for each planet in our sample. Furthermore, for all planets except HAT-P- $12 \mathrm{~b}, \mathrm{CH}_{4}$ is less abundant than $\mathrm{CO}$ on the dayside by at least 2-4 orders of magnitude. Comparing only the nightside profiles, we see that nightside $\mathrm{CH}_{4}$ abundance increases with decreasing $T_{e q}$, consistent with theoretical expectations. These nightside abundances, however, are still orders of magnitude smaller than nightside CO abundances for WASP-39b, HAT-P-1b, HD 209458b, WASP-31b, WASP-17b, and WASP-19b.

The dayside and nightside $\mathrm{CH}_{4}$ abundances are directly related to the trends in day-night temperature variation with increasing $T_{e q}$ (Figs. 1 and 5). WASP19b, with its large day-night temperature contrast, exhibits the largest differences in day-night $\mathrm{CH}_{4}$ abun- dances, with $\mathrm{CH}_{4}$ more than four orders of magnitude more abundant on the nightside than the dayside (bottom right panel). Conversely, for planets with small day-night temperature contrasts (e.g., WASP-39b, HAT$\mathrm{P}-12 \mathrm{~b}$ ), the differences in day-night $\mathrm{CH}_{4}$ abundance are much smaller. For HAT-P-12b, WASP-6b and HD $189733 \mathrm{~b}, \mathrm{CH}_{4}$ becomes nearly equal in abundance to $\mathrm{CO}$ on the nightside, particularly at pressures near 1 mbar. For HAT-P-12b, methane is actually more abundant than $\mathrm{CO}$ on the nightside at pressures less than 1 mbar.

The differences in $\mathrm{CO} / \mathrm{CH}_{4}$ abundance are more dramatic when considering the east and west terminators (Fig. 7). Differences between east- and west-limb $\mathrm{CH}_{4}$ abundances are largest for those with the largest differences in limb temperatures (see Fig 5). For the hottest planets in our sample (HAT-P-1b, HD 209458b, WASP$31 \mathrm{~b}$, WASP-17b, and WASP-19b), CO is the dominant carbon-bearing molecule on both the western and eastern limbs, particularly at the mbar pressures probed in transmission. However, for cooler planets (WASP-6b, WASP-39b, HD 189733b) while CO is more abundant 

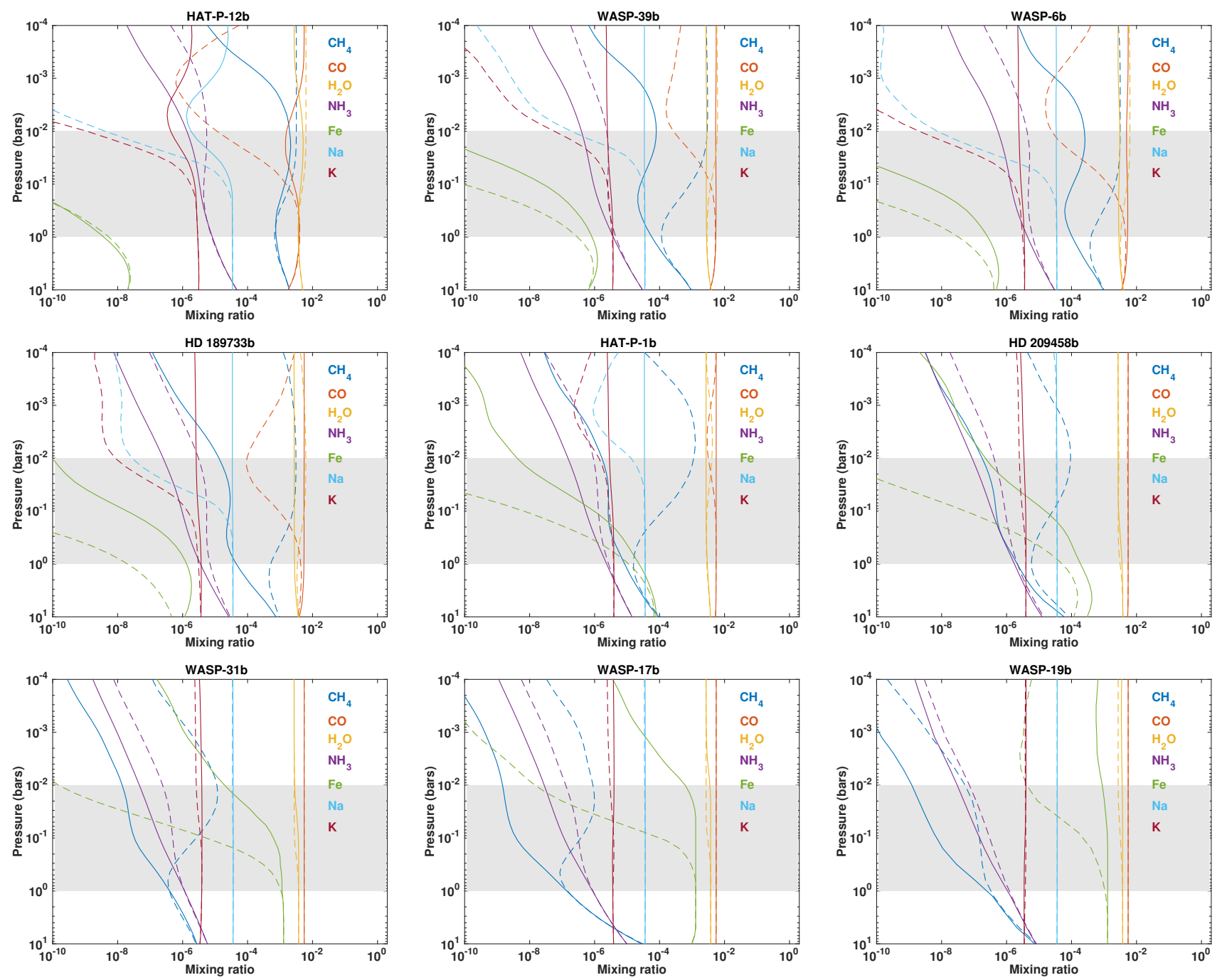

Figure 7. Chemical equilibrium abundances across the eastern (solid profiles) and western (dashed profiles) limbs for each LP planet. $\mathrm{CH}_{4}$ and $\mathrm{CO}$ abundances are indicated by the thicker orange and blue lines, respectively. Grey boxes indicate the pressures typically probed in transmission.

on the eastern limb by at least an order of magnitude, methane is more abundant than $\mathrm{CO}$ on the western limb by at least 1-2 orders of magnitude. In the case of HAT$\mathrm{P}-12 \mathrm{~b}$, the coolest planet in our sample, methane is more abundant than $\mathrm{CO}$ on the western limb by 3-4 orders of magnitude, and methane and $\mathrm{CO}$ have nearly equal abundances on the eastern limb. These large deviations in molecular abundances occur at pressures below 100 mbar.

However, it is likely that for all but the hottest planets in our sample (WASP-17b and WASP-19b), CO and $\mathrm{CH}_{4}$ abundances may be out of equilibrium on the dayside, nightside, and limbs (Cooper \& Showman 2006; Agúndez et al. 2012, 2014). Cooper \& Showman (2006) have shown that that for temperatures $\leq 2000 \mathrm{~K}$, the $\mathrm{CO} / \mathrm{CH}_{4}$ intraconversion timescale at these temperatures and pressures is long $\left(\gg 10^{5} \mathrm{~s}\right)$. Horizontal and vertical advective timescales are comparatively short, and therefore the dynamics will likely force the $\mathrm{CO}$ and $\mathrm{CH}_{4}$ abundances to be fixed with pressure.

Overall, the wide variations in temperature with longitude and latitude can imply significant differences in chemical abundances, particularly with regards to $\mathrm{Na}$, $\mathrm{K}, \mathrm{CO}$ and $\mathrm{CH}_{4}$ abundances. We discuss their significance further, as well as their observational prospects, in Section 4.

\subsection{Comparison to Spitzer eclipse data}

Using our nine-planet sample, we can look for trends not only in temperature and molecular abundances, but also how that translates to trends in observations. We focus primarily on observations in emission, as they are more directly related to each planet's thermal structure, and much less sensitive to the presence of clouds. We show theoretical dayside emission spectra generated from our circulation models in Figure 8. For all planets, absorption features due to $\mathrm{Na}, \mathrm{K}, \mathrm{CH}_{4}$ and $\mathrm{H}_{2} \mathrm{O}$ are apparent. These spectral features deepen as we probe the upper atmospheres of the cooler planets (compare, for example, the features for WASP-6b as compared to the features in WASP-17b). With its large mixing ratio, CO is expected to be abundant across the sample, but its spectral features are less prominent due to its smaller absorption cross section. 

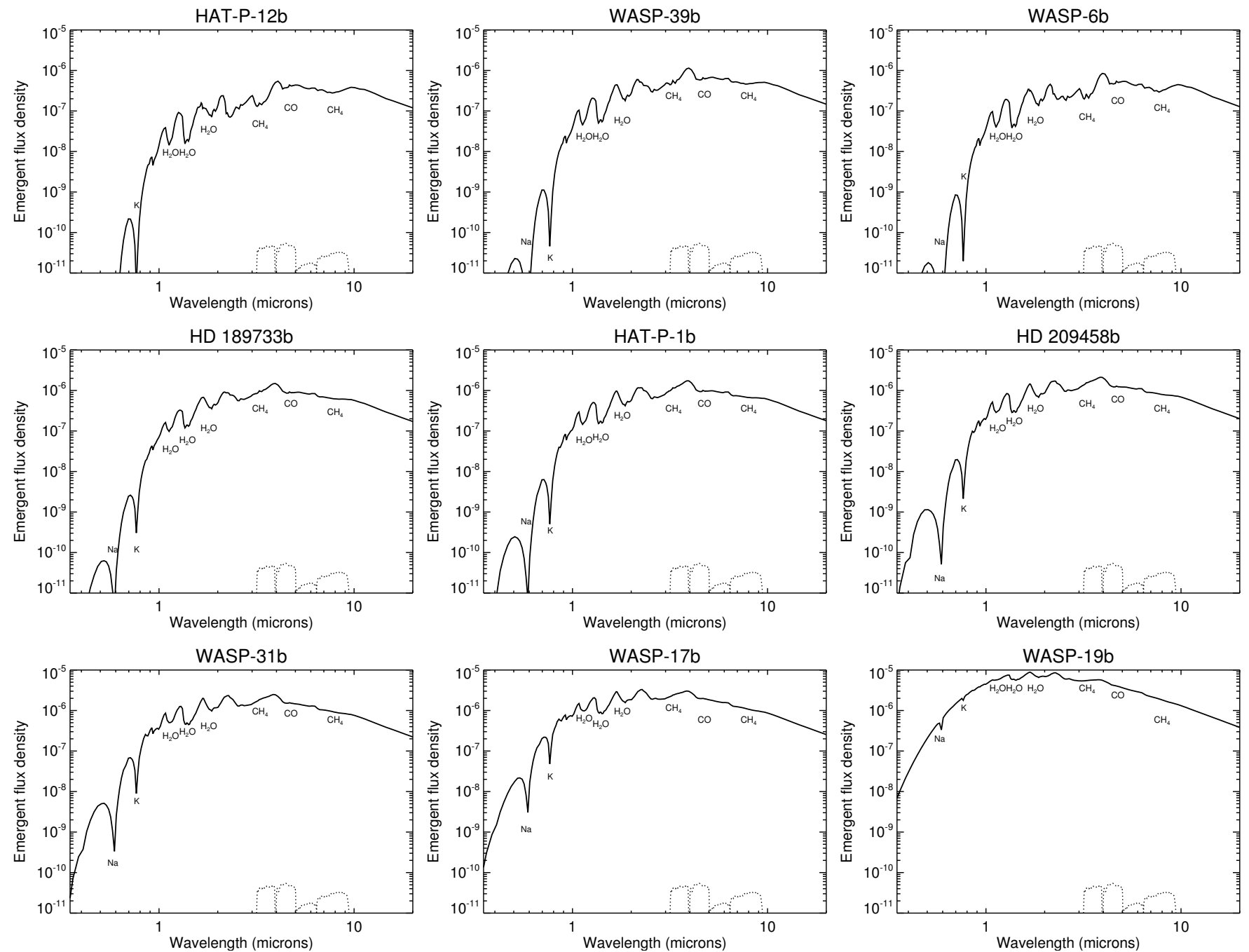

Figure 8. Dayside emergent flux density (ergs s $\mathrm{s}^{-1} \mathrm{~cm}^{-2} \mathrm{~Hz}^{-1}$ ) vs. wavelength for each LP target. Normalized transmission functions for Spitzer 3.6, 4.5, 5.8 and $8.0 \mu \mathrm{m}$ channels are shown in black dotted lines.

We can then compare our models to available secondary eclipse data by calculating the ratio of the planetary flux to the stellar flux for each system (Figure 9 ). The model spectra are plotted in pink profiles, with the binned fluxes for each Spitzer channel in pink dots. Transmission curves for each Spitzer channel are shown as black dotted curves. Overplotted in black dots with error bars are published Spitzer secondary eclipse depths for WASP-39b (Kammer et al. 2015), WASP-6b (Kammer et al. 2015), HD 1897433b (Charbonneau et al. 2008; Agol et al. 2010; Knutson et al. 2012), HAT-P-1b (Todorov et al. 2010), HD 209458b (Diamond-Lowe et al. 2014), WASP-17b (Anderson et al. 2011) and WASP-19b (Anderson et al. 2013).

Overall, each $1 \times$ solar model spectrum is in good general agreement with each Spitzer dataset. This is noteworthy for a number of reasons. First, while comparative circulation studies to date have only explored synthetic observations for one or two planets models over a wide phase space (e.g., Showman et al. 2009; Kataria et al. 2015; Showman et al. 2015), our study is the first to present synthetic observations from nine individual planet models, with their individual system parameters. Second, we generate theoretical observations from those models without any additional tuning (e.g., by varying drag, C/O ratio, chemistry, clouds, etc.). The fact that these purely three-dimensional outputs are in generally good agreement over over such a wide phase space is notable.

Still, there are discrepancies between the models and the observations, mostly notably the 5.8 and 8 micron points for HD $189733 \mathrm{~b}$, the 4.5 and 8 micron points for HAT-P-1b, and the 4.5 micron eclipse depth for WASP17b. In Figure 10, we quantify the agreement by differencing the observed and model eclipse depths for each Spitzer observation (black and pink points, respectively), and plot them as a function of $T_{e q}$. The variation of the 3.6, 4.5, 5.8 and $8.0 \mu \mathrm{m}$ points (red, green, navy, and light blue points, respectively) with $T_{e q}$ does not appear to follow any clear trend, suggesting that our model disagreement does not correlate with equilibrium temperature.

It is interesting to note that the points with the least agreement are those with the oldest reductions and some of the largest uncertainties (e.g., HAT-P-1b). We look forward to a re-analysis of these data to determine if revisions in eclipse depths are warranted, as has been the case for HD 189733b and HD 209458b (Knutson et al. 2012; 

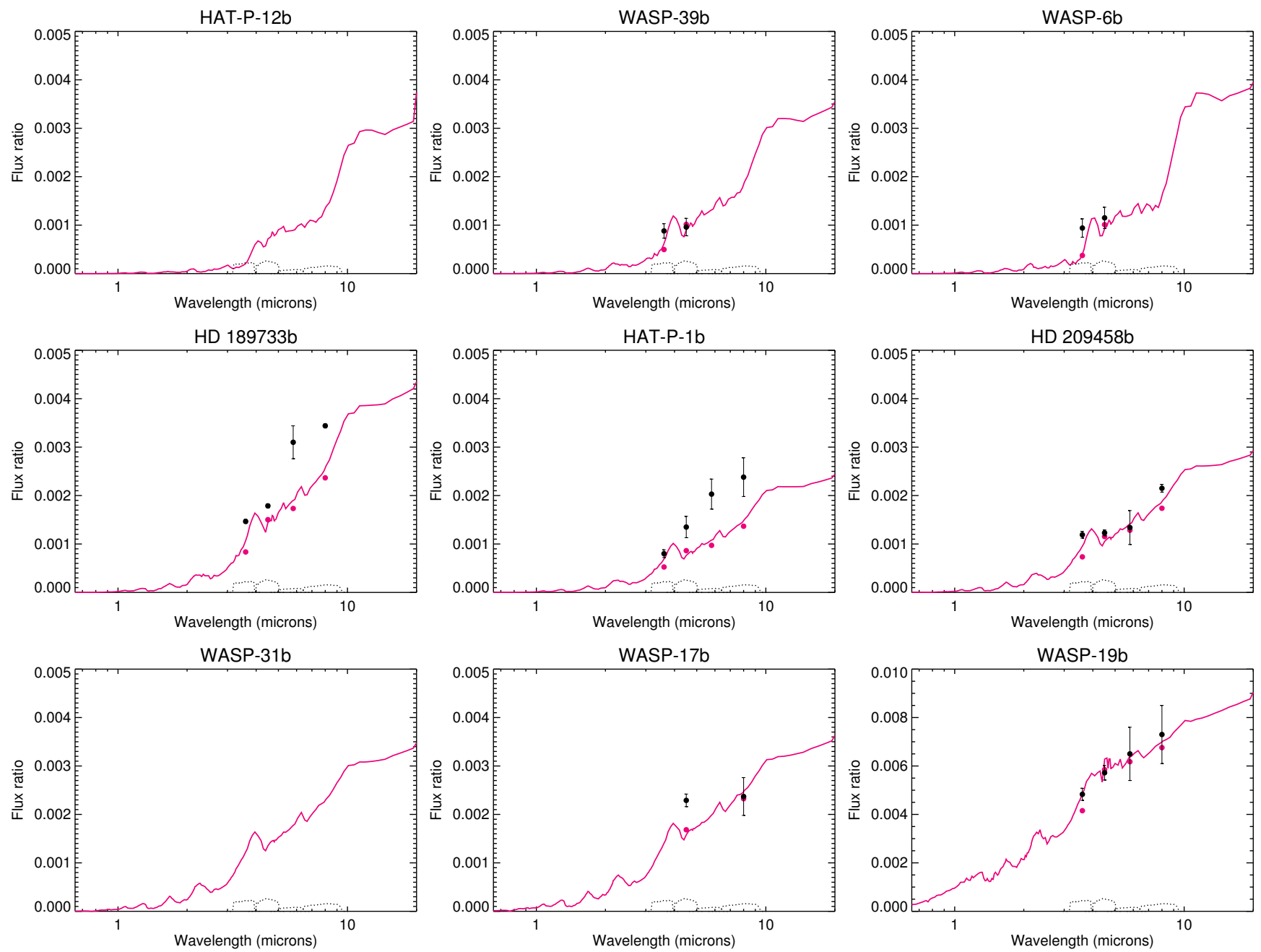

Figure 9. Planet-to-star flux ratio (pink profiles) vs. wavelength for each LP planet. Pink dots show the flux ratio integrated over each Spitzer bandpass, whose normalized transmission functions are shown as black dotted lines. Overplotted in black dots with error bars are published Spitzer secondary eclipse depths for WASP-39b, WASP-6b, HD 1897433b, HAT-P-1b, HD 209458b, WASP-17b and WASP-19b. See text for corresponding references to each dataset.

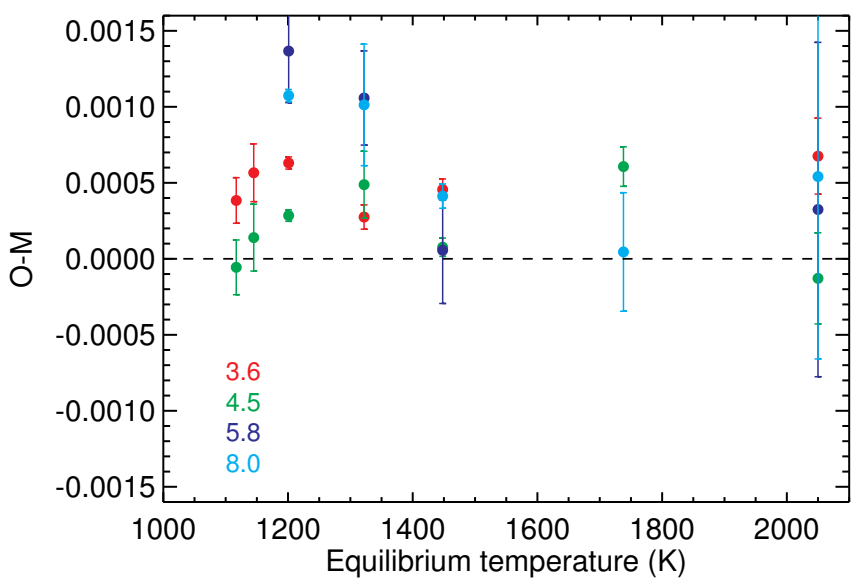

Figure 10. Difference in observed and model eclipse depths as a function of equilibrium temperature, $T_{e q}$. Red, green, navy and light blue points, with error bars, correspond to eclipse depth differences at 3.6, 4.5, 5.8 and $8.0 \mu \mathrm{m}$.

Diamond-Lowe et al. 2014; Zellem et al. 2014; Evans et al. 2015). Furthermore, our models seem to agree best with the $4.5 \mu \mathrm{m}$ points, where we are probing the abundance of $\mathrm{CO}$, which is not expected to vary widely with $T_{e q}$ (Figure 6).

Nevertheless, Figure 10 shows that our circulation models generally underpredict the dayside flux, although only on the $\sim 100 \mathrm{ppm}$ level (with uncertainties). This underprediction could be due to differences in atmospheric metallicity. For example, Knutson et al. (2012) show that the Spitzer dayside photometry of HD 189733b is well-matched by a model with an atmospheric composition of $5 \times$ solar. Furthermore, preliminary analyses of Spitzer phase curves of WASP-19b at 3.6 and $4.5 \mu \mathrm{m}$ show that an enhanced metallicity atmosphere (also $5 \times$ solar) is in better agreement with the amplitude of IR flux and timing of peak IR flux (Wong et al. 2015a). Magnetic drag or high $\mathrm{C} / \mathrm{O}$ ratios could also enhance dayside fluxes and provide better agreement.

\section{DISCUSSION}

Using the circulation models for our nine-planet sample, we have shown that over a wide range of planet radii, gravities, orbital periods, and equilibrium temperatures, equatorial superrotation continues to be a robust dynam- 
ical feature for hot Jupiters, and results in the eastward displacement of the dayside hotspot at photospheric pressures. Our results from Table 2 suggest that with increas$\operatorname{ing} T_{e q}$, hot Jupiters exhibit larger phase amplitudes and peak IR flux offsets that occur closer to secondary eclipse. While phase curve observations have only been collected for a few hot Jupiters (see Wong et al. 2015b, and references therein), we look forward to future phase curve observations with Spitzer, HST, K2, and JWST to further probe $T_{e q}$ space.

Although the focus of this study is to compare the planet sample in its entirety, we note that our models of HD 189733b and HD 209458b are qualitatively similar to circulation models by ours and other groups (e.g., Showman et al. 2009; Dobbs-Dixon et al. 2010; Heng et al. 2011; Dobbs-Dixon \& Agol 2013; Kataria et al. 2013; Liu \& Showman 2013; Mayne et al. 2013; Parmentier et al. 2013; Perna et al. 2010; Rauscher \& Menou 2013; Rogers \& Komacek 2014; Rogers \& Showman 2014). Each model exhibits equatorial superrotation with peak speeds on the order of $1 \mathrm{~km} \mathrm{~s}^{-1}$, and has an eastward-shifted $(\sim$ $\left.20-40^{\circ}\right)$ hotspot at photospheric pressures. The differences between models could arise in part from differences in the dynamical core: Dobbs-Dixon et al. (2010), DobbsDixon \& Agol (2013) and Mayne et al. (2013), for example, employ the fully compressible, 3D Navier-Stokes equations, while Rogers \& Komacek (2014) and Rogers \& Showman (2014) employ a 3D magnetohydrodnamics (MHD) model in the anelastic approximation. Differing radiative transfer schemes also lead to differences in heating and cooling and therefore day-night temperature contrasts and equatorial jet speeds; Dobbs-Dixon et al. (2010) and Rauscher \& Menou (2013) utilize a dualband radiative transfer model in modeling HD $189733 \mathrm{~b}$ and HD 209458b, while Heng et al. (2011), Mayne et al. (2013), Rogers \& Komacek (2014) and Rogers \& Showman (2014) use a Newtonian cooling scheme to model HD 209458b. Lastly, some models include the effects of either magnetic drag and/or ohmic dissipation, which serve to reduce day-night temperature contrasts and equatorial jet speeds (Perna et al. 2010; Rauscher \& Menou 2013; Rogers \& Komacek 2014; Rogers \& Showman 2014).

Our results in Section 3.3 demonstrate that one must be careful in extrapolating atmospheric chemical abundances based on a single observational technique. We cannot assume, for example, that the constrained abundances from an emission spectrum will be the same abundances as a transmission spectrum, even for cloud-free atmospheres, as each set of observations probes difference longitudes, pressures, and chemical regimes. Figures 6 and 7 show that these abundances can vary by many orders of magnitude, especially when comparing the abundances on the dayside and terminators. As the fidelity of exoplanet observations improve, one must be more careful to consider these factors particularly when combining data sets to constrain global thermal and chemical properties of a given planet.

High-dispersion $(R \equiv \lambda / \Delta \lambda \sim 100,000)$ spectrographs have the potential to probe the spatial chemical variations shown in our models. Already, these instruments have demonstrated the ability to detect and resolve $\mathrm{Na}$, $\mathrm{K}, \mathrm{CO}, \mathrm{CH}_{4}$ and $\mathrm{H}_{2} \mathrm{O}$ spectral lines for transiting hot Jupiters in transmission (Redfield et al. 2008; Snellen et al. 2008; Snellen et al. 2010; Wyttenbach et al. 2015;
Louden \& Wheatley 2015; Brogi et al. 2015), and for transiting and non-transiting hot Jupiters in emission (Rodler et al. 2012; Birkby et al. 2013; de Kok et al. 2013; Brogi et al. 2012, 2013, 2014; Schwarz et al. 2015). Future high-resolution spectrographs such as ESPRESSO or CRIRES+ on the Very Large Telescope (VLT), or HiReS and METIS on the Extremely Extra Large Telescope (E-ELT), would be well-suited to this task. The JWST/MIRI and NIRSpec instruments could potentially probe and resolve these spectral variations as well.

Such measurements could focus solely on $\mathrm{Na}$ and $\mathrm{K}$, as has already been done for HD 189733b and HD 209458b (e.g., Redfield et al. 2008; Snellen et al. 2008; Wyttenbach et al. 2015; Louden \& Wheatley 2015). We would expect that for the cooler planets in our sample, $\mathrm{Na}$ and $\mathrm{K}$ would be less abundant on the western limb and nightside, which would translate to a smaller transmission signal. However, it should be noted that other Na- and Kbearing species become increasingly abundant at lower temperatures, such as $\mathrm{NaCl}, \mathrm{NaOH}$ and $\mathrm{KOH}$ (Lodders 1999). While monatomic Na will tend to dominate over most conditions, monatomic $\mathrm{K}$ may be depleted by the formation of these species. Furthermore, it is possible that cold traps (e.g., Parmentier et al. 2013) could also suppress variations in $\mathrm{Na}, \mathrm{K}$, and $\mathrm{Fe}$ abundance.

HAT-P-12b could be an ideal testbed for probing differences in $\mathrm{CO} / \mathrm{CH}_{4}$ abundances, as the planet could exhibit appreciable differences in these abundances between the east and west limbs, and could have equal abundances on the dayside, while also having a favorable brightness contrast compared to smaller, dimmer subNeptunes and super Earths. However, as noted earlier, it is possible that these carbon-bearing species would be out of equilibrium, which would freeze their abundances across the dayside, nightside and limbs. In this case, the differences in $\mathrm{CO}$ and $\mathrm{CH}_{4}$ abundance would be largely reduced (Agúndez et al. 2012, 2014; Cooper \& Showman 2006). We look forward to future observations to test these predictions.

The interpretation of these future datasets could also be complicated by the formation of clouds, as evidenced by our sample of transmission spectra (Sing et al. 2016). Line \& Parmentier (2015) have shown that partially cloudy limbs can appear as a residual in transit ingress and egress with a residual of $\sim 100 \mathrm{ppm}$. While we do not compare our cloud-free models directly to transmission spectra to interpret their cloud properties, we can use our model results to explore their potential threedimensional structure. If we first consider the limbaveraged temperature maps in Figure 4, it is likely that a particular cloud species could form high in the atmosphere at the equator and mid-latitudes, while forming at deeper pressures at high latitudes. These clouds will contribute equally to a transmission spectrum.

We can also use Figure 5 to further comment on cloud properties, as each panel includes condensation curves for chemical species expected to condense in the atmospheres of our planet sample (grey dotted profiles). Intersections between each condensation curve and $p$-T profile indicate potential cloud bases for that particular species. While temperature differences between each $p$ - $\mathrm{T}$ profile can be small $(\sim 50-75 \mathrm{~K})$, these small changes can still imply large differences in cloud properties. For example, the hot Jupiters HAT-P-12b, WASP-6b and HD 189733b 
have temperatures that could allow for the formation of $\mathrm{ZnS}, \mathrm{KCl}$ and $\mathrm{Na}_{2} \mathrm{~S}$ clouds at observable pressures, but only on the western limb and nightside (green and yellow profiles). This is notable, as transmission spectra for all three targets suggest their atmospheres are cloudy. Western limb clouds are similar to the scenario proposed for hot Jupiter Kepler-7b, which has been postulated to have clouds westward of its substellar point based on Spitzer and Kepler phase curves (Demory et al. 2013). Also, nightside condensation has long been hypothesized for hot Jupiters and brown dwarfs, particularly as a means for removing $\mathrm{Na}$ via $\mathrm{Na}_{2} \mathrm{~S}$ condensation (Visscher et al. 2006 , and references therein). Finally, all five $p$-T profiles of WASP-31b cross numerous condensation curves at observable pressures, including those for silicate clouds. This suggests that its atmosphere can host many types of clouds, consistent with its cloudy transmission spectrum.

It is interesting to note that HD $189733 \mathrm{~b}$, WASP-39b and WASP-6b appear to have very similar wind and temperature structures, which yield similar variations in molecular abundances and emission spectra. However, within the LP transmission spectral survey, HD 189733b and WASP-6b appear cloudy, while WASP-39b appears comparatively clear (Sing et al. 2015). Overall, because these models are cloud-free, more physics is warranted to further explain the trends seen in the transmission spectra. Detailed cloud models would account for variations in horizontal and vertical mixing, which could allow for the transport of cloud particles at depth or at varying longitudes. High-altitude photochemical hazes are also likely to be important, as they form at temperatures less than or equal to $1000-1100 \mathrm{~K}$ (Zahnle et al. 2009; Moses 2014). We save a detailed exploration of cloud properties for a future paper (Kataria et al., in preparation).

\section{CONCLUSIONS}

We present three-dimensional circulation models for a "grid" of nine hot Jupiters that comprise a transmission spectral survey using the Hubble and Spitzer Space Telescopes, the largest circulation comparison study conducted for hot Jupiters to date. We utilize these models as a first step to interpreting their transmission spectra, which imply a large diversity in clouds and haze properties.

Across the wide range of parameters for each individual system, we show that each planet atmosphere exhibits equatorial superrotation, with eastward displacement of the hottest regions from the substellar longitude. We also show that variations in temperature, particularly from the dayside to nightside, and across the western and eastern limbs, can produce large variations in chemistry. These temperature variations could also imply large variations in cloud properties across those regions, though we do not investigate those aspects in detail here.

Transmission observations have the greatest potential for probing these chemical variations, especially with the high-resolution spectrographs aboard the next generation of telescopic facilities (e.g., VLT, E-ELT, JWST). Indeed, even current RV instruments such as the HARPS spectrograph have demonstrated the potential to probe variations in $\mathrm{Na}$ and $\mathrm{K}$ abundances (Wyttenbach et al. 2015; Louden \& Wheatley 2015).

In comparing synthetic emission spectra for each planet with available Spitzer eclipse observations, we find that while our solar metallicity, drag and cloud-free models agree reasonably well to observations, they do systematically underpredict the measured dayside fluxes. This could point to enhanced metallicity or drag, which would help to increase the day/night temperature contrast and therefore the dayside emergent flux. If such scenarios are the case, that could also imply the abundance variations discussed here would be further amplified. In summary, the hot Jupiters continue to be an exoplanet population rife for characterization and further understanding. The lessons we learn about their chemistry and clouds are valuable for informing future observations of Neptunes, super Earths, and habitable exoplanets.

We thank Caroline Morley, Nikolay Nikolov, Thomas Evans and Hannah Wakeford for useful discussions which improved the manuscript. We also acknowledge that part of this work was completed at the Space Telescope Science Institute (STScI) operated by AURA, Inc. This work is based on observations with the NASA/ESA HST, obtained at the STScI. This work is also based in part on observations made with the Spitzer Space Telescope, which is operated by the Jet Propulsion Laboratory, California Institute of Technology under a contract with NASA. The research leading to these results has received funding from the European Research Council under the European Unions Seventh Framework Program (FP7/2007-2013) / ERC grant agreement no. 336792. Support for this work was provided by NASA through grants under the HST-GO-12473 program from the STScI. This work used the DiRAC Complexity system, operated by the University of Leicester IT Services, which forms part of the STFC DiRAC HPC Facility (www.dirac.ac.uk). This equipment is funded by BIS National E-Infrastructure capital grant ST/K000373/1 and STFC DiRAC Operations grant ST/K0003259/1. DiRAC is part of the National E-Infrastructure. We also thank the anonymous referee for comments that greatly improved the paper.

\section{REFERENCES}

Adcroft, A., Campin, J.-M., Hill, C., \& Marshall, J. 2004, Monthly Weather Review, 132, 2845

Agol, E., Cowan, N. B., Knutson, H. A., et al. 2010, ApJ, 721, 1861

Agúndez, M., Parmentier, V., Venot, O., Hersant, F., \& Selsis, F. 2014, Astronomy and Astrophysics, 564, A73

Agúndez, M., Venot, O., Iro, N., et al. 2012, Astronomy and Astrophysics, 548, A73

Anderson, D. R., Smith, A. M. S., Lanotte, A. A., et al. 2011, MNRAS, 416, 2108

Anderson, D. R., Smith, A. M. S., Madhusudhan, N., et al. 2013, MNRAS, 430, 3422

Arakawa, A., \& Lamb, V. R. 1977, Methods of Computational Physics, 17, 173

Birkby, J. L., de Kok, R. J., Brogi, M., et al. 2013, MNRAS, 436, L35

Brogi, M., de Kok, R. J., Albrecht, S., et al. 2015, ArXiv e-prints Brogi, M., de Kok, R. J., Birkby, J. L., Schwarz, H., \& Snellen, I. A. G. 2014, A\&A, 565, A124

Brogi, M., Snellen, I. A. G., de Kok, R. J., et al. 2012, Nature, 486, 502

- 2013, ApJ, 767, 27

Charbonneau, D., Brown, T. M., Noyes, R. W., \& Gilliland, R. L. 2002, ApJ, 568, 377

Charbonneau, D., Knutson, H. A., Barman, T., et al. 2008, ApJ, 686, 1341 
Cooper, C. S., \& Showman, A. P. 2006, ApJ, 649, 1048

de Kok, R. J., Brogi, M., Snellen, I. A. G., et al. 2013, A\&A, 554, A82

de Wit, J., Gillon, M., Demory, B.-O., \& Seager, S. 2012, A\&A, 548, A128

Deming, D., Brown, T. M., Charbonneau, D., Harrington, J., \& Richardson, L. J. 2005, ApJ, 622, 1149

Demory, B.-O., de Wit, J., Lewis, N., et al. 2013, ApJ, 776, L25

Diamond-Lowe, H., Stevenson, K. B., Bean, J. L., Line, M. R., \& Fortney, J. J. 2014, ApJ, 796, 66

Dobbs-Dixon, I., \& Agol, E. 2013, Monthly Notices of the Royal Astronomical Society, 435, 3159

Dobbs-Dixon, I., Cumming, A., \& Lin, D. N. C. 2010, The Astrophysical Journal, 710, 1395

Evans, T. M., Aigrain, S., Gibson, N., et al. 2015, MNRAS, 451, 680

Goody, R., West, R., Chen, L., \& Crisp, D. 1989, J. Quant. Spectrosc. Radiative Transfer, 42, 539

Hauschildt, P. H., Allard, F., \& Baron, E. 1999, ApJ, 512, 377

Heng, K., Menou, K., \& Phillipps, P. J. 2011, Monthly Notices of the Royal Astronomical Society, 413, 2380

Holton, J. R. 1992, An introduction to dynamic meteorology

Huitson, C. M., Sing, D. K., Pont, F., et al. 2013, MNRAS, 434, 3252

Kammer, J. A., Knutson, H. A., Line, M. R., et al. 2015, ApJ, 810,118

Kataria, T., Showman, A. P., Fortney, J. J., Marley, M. S., \& Freedman, R. S. 2014, ApJ, 785, 92

Kataria, T., Showman, A. P., Fortney, J. J., et al. 2015, ApJ, 801,86

Kataria, T., Showman, A. P., Lewis, N. K., et al. 2013, Astrophysical Journal, 767, 76

Knutson, H. A., Lewis, N., Fortney, J. J., et al. 2012, Astrophysical Journal, 754, 22

Komacek, T. D., \& Showman, A. P. 2016, arXiv.org

Lewis, N. K., Showman, A. P., Fortney, J. J., Knutson, H. A., \& Marley, M. S. 2014, ApJ, 795, 150

Lewis, N. K., Showman, A. P., Fortney, J. J., et al. 2010, Astrophysical Journal, 720, 344

Line, M. R., \& Parmentier, V. 2015, arXiv.org, 9443

Liu, B., \& Showman, A. P. 2013, ApJ, 770, 42

Lodders, K. 1999, The Astrophysical Journal, 519, 793

Lodders, K. 2003, ApJ, 591, 1220

Louden, T., \& Wheatley, P. J. 2015, ApJ, 814, L24

Marley, M. S., \& McKay, C. P. 1999, Icarus, 138, 268

Mayne, N. J., Baraffe, I., Acreman, D. M., et al. 2013, Astronomy and Astrophysics, 561, A1

Moses, J. I. 2014, Philosophical Transactions of the Royal Society A: Mathematical, Physical and Engineering Sciences, 372, 20130073

Nikolov, N., \& Sainsbury-Martinez, F. 2015, ApJ, 808, 57

Nikolov, N., Sing, D. K., Pont, F., et al. 2014, MNRAS, 437, 46

Nikolov, N., Sing, D. K., Burrows, A. S., et al. 2015, MNRAS, 447,463
Parmentier, V., Showman, A. P., \& Lian, Y. 2013, Astronomy and Astrophysics, 558, A91

Perez-Becker, D., \& Showman, A. P. 2013, ApJ, 776, 134

Perna, R., Menou, K., \& Rauscher, E. 2010, The Astrophysical Journal, 719, 1421

Pont, F., Sing, D. K., Gibson, N. P., et al. 2013, MNRAS, 432 , 2917

Rauscher, E., \& Menou, K. 2013, The Astrophysical Journal, 764, 103

Redfield, S., Endl, M., Cochran, W. D., \& Koesterke, L. 2008, ApJ, 673, L87

Rodler, F., Lopez-Morales, M., \& Ribas, I. 2012, ApJ, 753, L25

Rogers, T. M., \& Komacek, T. D. 2014, The Astrophysical Journal, 794, 132

Rogers, T. M., \& Showman, A. P. 2014, The Astrophysical Journal, 782, L4

Schwarz, H., Brogi, M., de Kok, R., Birkby, J., \& Snellen, I. 2015, A\&A, 576, A111

Showman, A. P., Cho, J. Y.-K., \& Menou, K. 2010, Atmospheric Circulation of Exoplanets, ed. S. Seager, 471-516

Showman, A. P., Fortney, J. J., Lewis, N. K., \& Shabram, M. 2013, Astrophysical Journal, 762, 24

Showman, A. P., Fortney, J. J., Lian, Y., et al. 2009, Astrophysical Journal, 699, 564

Showman, A. P., Lewis, N. K., \& Fortney, J. J. 2015, ApJ, 801, 95

Showman, A. P., \& Polvani, L. M. 2011, ApJ, 738, 71

Sing, D. K., Lecavelier des Etangs, A., Fortney, J. J., et al. 2013, MNRAS, 436, 2956

Sing, D. K., Wakeford, H. R., Showman, A. P., et al. 2015, MNRAS, 446, 2428

Sing, D. K., Fortney, J. J., Nikolov, N., et al. 2016, Nature, 529, 59

Snellen, I. A. G., Albrecht, S., de Mooij, E. J. W., \& Le Poole, R. S. 2008, A\&A, 487, 357

Snellen, I. A. G., de Kok, R. J., de Mooij, E. J. W., \& Albrecht, S. 2010, Nature, 465, 1049

Todorov, K., Deming, D., Harrington, J., et al. 2010, ApJ, 708, 498

Visscher, C., Lodders, K., \& Fegley, B. J. 2006, The Astrophysical Journal, 648, 1181

Wakeford, H. R., Sing, D. K., Deming, D., et al. 2013, MNRAS, 435, 3481

Wong, I., Knutson, H. A., Kataria, T., et al. 2015a, ArXiv e-prints

Wong, I., Knutson, H. A., Lewis, N. K., et al. 2015b, ApJ, 811, 122

Wyttenbach, A., Ehrenreich, D., Lovis, C., Udry, S., \& Pepe, F. 2015, A\&A, 577, A62

Zahnle, K., Marley, M. S., \& Fortney, J. J. 2009, arXiv.org

Zellem, R. T., Lewis, N. K., Knutson, H. A., et al. 2014 Astrophysical Journal, 790, 53 